\title{
A mosaic renal myeloid subtype with T-cell inhibitory and protumoral features is linked to immune escape and survival in clear cell renal cell cancer
}

Dorothee Brech ${ }^{1}$, Tobias Straub², Evangelos Kokolakis ${ }^{1}$, Martin Irmler ${ }^{3}$, Johannes Beckers ${ }^{3,4,5}$, Florian Buettner6,7, Elke Schaeffeler ${ }^{6,7}$, Stefan Winter ${ }^{6,7}$, Matthias Schwab $6,7,8,9$, Peter J. Nelson ${ }^{10}$ and Elfriede Noessner ${ }^{1 *}$

1 Immunoanalytics/Tissue Control of Immunocytes, Helmholtz Zentrum München, Neuherberg, Germany

${ }^{2}$ Bioinformatics Core Unit, Biomedical Center, Ludwig-Maximilians-University, Munich, Germany

3 Institute of Experimental Genetics, Helmholtz Zentrum München, Neuherberg, Germany

${ }^{4}$ German Center for Diabetes Research (DZD), Munich, Germany

${ }^{5}$ Chair of Experimental Genetics, Technical University of Munich, Freising, Germany

${ }^{6}$ Dr. Margarete Fischer-Bosch-Institute of Clinical Pharmacology, Stuttgart, Germany;

${ }^{7}$ University of Tuebingen, Tuebingen, Germany

8 Department of Clinical Pharmacology and Department of Pharmacy and Biochemistry, University of Tuebingen, Tuebingen, Germany.

${ }^{9}$ German Cancer Consortium (DKTK) and German Cancer Research Center (DKFZ), Heidelberg, Tübingen, Germany

10 Medizinische Klinik und Poliklinik IV, University of Munich, Munich, Germany

*Correspondence: noessner@helmholtz-muenchen.de. 
Keywords: mononuclear phagocyte system, macrophage plasticity, tissue macrophage, tumor microenvironment, gene expression, immune tolerance, immunotherapy, renal cell carcinoma

\section{Highlights}

Bullet points:

- Renal cell carcinoma (ccRCC) harbors polarized mosaic myeloid cells (ercDCs)

- ercDCs are found in contact with dysfunctional T cells in ccRCC

- ercDCs express novel immunoinhibitory proteins

- High ercDC z-score in ccRCC tissue correlates with poor patient survival 


\section{Summary}

Mononuclear phagocytes moderate tissue repair, immune activation and tolerance. In the renal tubulo-interstitium specialized dendritic cells help maintain homeostasis and protect tubuli from immune injury. Human renal cell carcinoma (RCC) is immunogenic;

yet immunotherapies that target T-cell dysfunction show limited clinical efficacy suggesting additional mechanisms of immunoinhibiton. We previously described "enriched-in-renal cell carcinoma" (erc)DCs that are often found in tight contact with T cells which are dysfunctional. Here we describe that ercDCs exhibit a distinct polarization state imparted by tissue-specific signals characteristic for RCC and renal tissue homeostasis. The resulting mosaic transcript signature includes features associated with host defense activity, angiogenesis/invasion and T-cell inhibition. An ercDC-specific profile was predictive for patient survival and suggests potential therapeutic targets for improved immunotherapy. 


\section{Significance}

Immunotherapies, which re-invigorate T-cell activity, achieve clinical responses in subsets of patients only revealing additional layers of T-cell inhibition. Mononuclear phagocytes can be immunoinhibitory. But, they are highly plastic and repolarization may be possible if key programming molecules can be identified, potentially enabling antitumor responses in tumors refractory to checkpoint blockade. We describe a myeloid cell type with mosaic feature including tumor-promotion and immunoinhibition in human clear cell renal cell carcinoma. Observed tight contacts with T cells may translate into T-cell dysfunction. A high ercDC score in tumor tissue correlates with poor patient survival suggesting ercDCs as targets for therapeutic intervention. Targeting molecules that are identified in the ercDC profile may expand the range of patients effectively treated by immunotherapy. 


\section{Introduction}

Dendritic cells (DCs) and macrophages of the mononuclear phagocyte system (MPS) are central players in the control of tissue homeostasis, wound healing and damage prevention. These processes are mediated in part through their capacity to remove cellular debris and induce angiogenesis, as well as by their ability to activate or induce tolerization of relevant immunocytes. The effector activities of MPS cells are strongly dependent on the cell's polarization state which is imprinted by cues from the local environment (Das et al., 2015; Davies et al., 2013; Gordon et al., 2014).

In the murine kidney, the tubulointerstitial region is densely covered by myeloid cells expressing the kidney-specific homing receptor $\mathrm{CX}_{3} \mathrm{CR} 1$ (Engel et al., 2015; Hochheiser and Kurts, 2015; Soos et al., 2006). Due to their stellate morphology, these cells were designated DCs despite their dual expression of macrophage (F4/80) and DC markers (CD11c for murine DCs). The cells are thought to act as sentinels maintaining homeostasis and protecting the renal tubuli from immune-induced injury through tolerogenic mechanisms (Scholz et al., 2008). Clear cell renal cell carcinoma (ccRCC) arises from epithelial cells of the renal tubulointerstitium. This tumor type is generally richly infiltrated by immune cells, including T, NK and myeloid cells. However, despite abundance of $C D 8^{+} \mathrm{T}$ cells that can recognize and destroy tumor cells when taken out of the ccRCC environment (Jantzer and Schendel, 1998; Leisegang et al., 2010), control of tumor progression fails, suggesting local suppression of T-cell effector activity (Giraldo et al., 2015; Giraldo et al., 2017; Prinz et al., 2012). Invigorating the Tcell response through blockade of the immune checkpoint molecule PD-1 has been approved as $2^{\text {nd }}$ line treatment for metastatic RCC (Carlo et al., 2016; Motzer et al., 2015), but only a fraction of patients have been shown to respond. This suggests that mechanisms beyond those directly targeting the $\mathrm{T}$ cells help control the antitumor response, thus underscoring the critical need to better understand the tumor 
environment and to identify additional therapeutic targets beyond PD-1 to improve response rates and expand the range of patients that can be effectively treated.

We have previously shown enrichment of an unusual myeloid cells in patient ccRCC tissue that coexpress macrophage markers (CD14) and DC markers, i.e. CD209, which identifies them as a subset of interstitial DCs with crosspresentation ability (van Kooyk et al., 2013), as well as the costimulatory molecules HLA-DR and CD40. We designated the myeloid subpopulation "enriched-in-renal-carcinoma DCs" (ercDCs), due to its strong enrichment in the tumor center, lower abundance in the tumor periphery and even less presence in the non tumor-inflicted kidney area (Figel et al., 2011). We speculated that, analogous to the cell type described in murine kidney, ercDCs may also convey tolerogenic, tissue protective functions that shelter emerging tumor cells from immune attack. In ccRCC tissues, ercDCs were often found tightly engaged with T cells suggesting intercellular communication in situ (Figel et al., 2011). Through these observed T-cell contacts, we hypothesized that ercDCs could play a role in the suppressed T-cell function previously described (Prinz et al., 2012).

To help clarify the functional attributes of ercDCs and to position them within the MPS continuum, an analysis of the native cell population isolated from ccRCC tissue was performed. Transcriptomic profiling has been useful in defining subsets and polarization states within the MPS continuum (Hume and Freeman, 2014) and it has helped assign functional characteristics as demonstrated e.g. by Houser et al. who described two distinct subsets of $\mathrm{CD}^{+} 4^{+}$decidual marcophages, $\mathrm{CD} 11 \mathrm{c}^{\mathrm{HI}}$ and CD11 $\mathrm{C}^{\mathrm{LO}}$, with distinct functions in tissue remodeling, growth and development (Houser et al., 2011).

In the present study we provide a molecular characterization of ercDCs isolated from ccRCC tissues. A definition of functional characteristics as well as their relationship to myeloid subtypes from other human tissues was established. The transcriptomic 
profiling identified ercDCs as a unique myeloid subset within the macrophage spectrum, and placed them in close relationship to inflammatory macrophages from the ascites of human ovarian cancer. The identified ercDC-specific gene expression profile was predictive for patient survival and suggests potential targets for therapeutic intervention that may help improve clinical efficacy of immunotherapy. 


\section{Results}

The ercDC transcriptional profile identifies them as a unique myeloid subset within the macrophage spectrum

We recently reported that human $\mathrm{cCRCC}$ tissue harbor an unusual myeloid type, called ercDCs. ErcDCs resemble classical DCs by expression of costimulatory molecules (CD80, CD86, CD40), HLA-DR (Figure S1), and by their capacity to cross-present antigen. However, they also express both DC (CD209) and macrophage markers (CD14, CD163) (Figel et al., 2011). Here we report expression of additional surface markers that further highlight the unusual differentiation of this resident cell type (Figure S1). ErcDCs express CD141, a marker for crosspresenting myeloid cells, and $\mathrm{CX}_{3} \mathrm{CR} 1$, a homing receptor thought to regulate the abundance of DCs in the kidney (Hochheiser and Kurts, 2015, Hochheiser et al., 2013). Lack of CD83 and marginal levels of CCR7, but high levels of CCR1, CCR2 and CCR5 indicate an immature phenotype. Surface expression of other common myeloid markers, including CD68 and CD11b, is seen. In previous work we reported that ercDCs are tightly engaged with $T$ cells in ccRCC tissue (Figel et al., 2011) and that the tumor-infiltrating T cells (TILs) are dysfunctional with features of cell cycle arrest and suppressed AKT/ERK pathway activation (Prinz et al., 2012). ErcDCs were also found to secret the matrix metalloproteinases, MMP2 and MMP9 as well as TNF (Figel et al., 2011), suggesting tumor-promoting characteristics in addition to direct effects on immune cells. To obtain a deeper functional understanding of ercDCs, in particular as it relates to a potential involvement in shaping the antitumor immune response, we performed an unbaised analysis.

$\mathrm{CD}_{14}{ }^{+} \mathrm{CD} 209^{+}$ercDCs and CD209-CD14 ${ }^{+}$macrophages were sorted from $\mathrm{ccRCC}$ tissue cell suspensions (Figure S2A, tables S1 and S2A) using flow cytometry and subjected to genome-wide gene expression analysis. Reference transcriptomes were 
generated from sorted blood monocytes $\left(C D 14^{+}\right)$, slanDC and $C D 1 c^{+} D C$ (all from PBMCs of healthy donors (HD)), and from in vitro-polarized M1- and M2-macrophages as described (Murray et al., 2014; Martinez et al., 2006) (Figure S2B, table S2A).

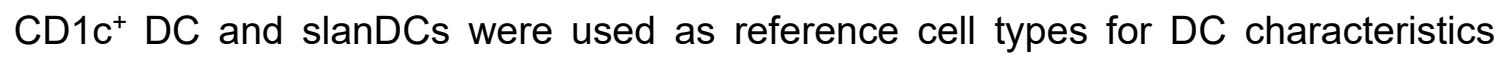
(Hansel et al., 2011; Nizzoli et al., 2013) to substitute for interstitial DCs, i.e. CD209+CD14- cells, which could not be sorted from ccRCC tissue cell suspensions due to low cell frequency.

Previously, using FACS analysis, it was difficult to assign ercDCs to either macrophages or DC subgroups as they co-expressed markers of both cell types, i.e. CD209, CD14, and co-stimulatory molecules CD80, CD86 and CD40. Analysis of their transcriptome with respect to core macrophage and DC genes (Gautier et al., 2012; Miller et al., 2012; Xue et al., 2014) now reveals that ercDC_ccRCC strongly express most of the human macrophage-associated core genes and show some expression of the established DC-associated core genes (Figure 1A). Protein expression analysis of selected macrophage (CD64A, CD14, CD32A, MerTK) and DC markers (ANPEP/CD13, FLT3) was confirmed by flow cytometry (Figure 1B, C). FLT3 or ANPEP/CD13, which are suggested DC-associated proteins (Xue et al., 2014), were not reliable for $\mathrm{DC} /$ macrophage distinction in our system (Figure $1 \mathrm{C}$ ). For example, FLT3 discriminated between DCs and macrophages on the transcript level, but strong surface protein expression was seen on all cell types, CD1 ${ }^{+} \mathrm{DCs}, \mathrm{M} 1-$ and $\mathrm{M} 2-$ macrophages, and also ercDCs.

A second MPS classification scheme based on transcription factors and growth factor receptors (Guilliams et al., 2014) showed robust expression of the macrophageassociated factors MAF, MAFB, CREG1 and CSF1R by ercDC_RCC. CSF1R protein expression was validated by flow cytometry (Figure 2A). In contrast, ercDCs weakly expressed the DC-associated transcription factors IKZF1, BCL6, IRF4 and the growth 
factor receptor FLT3 (Figure 2B). Thus, using two different means of classification, ercDCs appeared to more closely represent a macrophage rather than a DC subtype. Macrophages represent a contiuum of different subtypes, wherein M1- (classical) and M2- (alternatively activated) macrophages are positioned at the opposing ends of the polarization spectrum (Mantovani et al., 2004; Sica and Mantovani, 2012). However, tissue macrophages are extremely heterogeneous and may adopt specialized functions as they respond to a variety of signals that change during homeostasis and inflammation (Gordon et al., 2014; Gordon and Taylor, 2005). A classification system based on the fundamental homeostatic macrophage activities - host defense, wound healing/tissue modulation and immunoregulation - has been established to help address this complexity (Mosser and Edwards, 2008). To position the ercDC_ccRCC within these classification schemes, we analyzed the transcriptome with regards to gene lists associated with biologic function (Gustafsson et al., 2008; Mosser and Edwards, 2008; Wang et al., 2012) (tables S3, S4, S5) including also an invasive signature gene list (Wang et al., 2012) which we supplemented with key angiogenic genes taken from the GSEA MSigDB database (table S6). The ercDC_ccRCC shared expression patterns with both M1- and M2-macrophages (Figure 2C, table S3, S4). Notable was the strong expression of the prototypic M1 gene CD64A (Mantovani and Sica, 2010; Tarique et al., 2015). However, ercDC_ccRCC also shared many markers with M2-macrophages, including MerTK, CD204, CD206 and CD36. Validation of surface expression was performed for the M1-marker CD64A (Figure 1B) and M2markers (CD204, CD206, CD36) (Figure 2C). Single cell flow cytometry using ccRCC tissue suspensions confirmed on protein level that the majority of CD $14^{+}$CD209+ cells co-expressed CD64A (M1-marker) with MerTK and MSR1/CD204 (both M2-markers) (Figure 2D) supporting the suggestion that ercDCs might represent mosaic cells on the single cell level. 
ErcDC_ccRCC were found to express a number of chemokines (CXCL9, CXCL10, and CXCL11) that are characteristic of macrophage subtypes associated with host defense activity and help in the recruitment of Th1-polarized immune cells (Figure 2E). ErcDC_ccRCC were found to strongly express genes linked to wound healing and tissue remodeling (i.e. STAB1, FN1, F13A) (Figure 2F), thus resembling M2-MФ_in vitro and CD11 $\mathrm{C}^{\mathrm{LO}} \mathrm{M \Phi} \_$decidua, which have been linked to wound healing and tissue remodeling (Martinez et al., 2009; Martinez et al., 2008; Zhang and Mosser, 2008; Houser et al., 2011). The ercDC transcriptome appeared more similar to M2macrophages with regards to immunoregulatory genes (i.e. MAF, VSIG4, TREM2, CD206) (Figure 2G) (Mosser, 2003; Pollard, 2009; Morris et al., 2011). In addition, ercDCs showed a strong signature of angiogenesis and invasion-associated genes. These included genes associated with the recruitment of proinflammatory monocytes, e.g. CCL2, CCL8 and NRP1, as well as proinflammatory factors such as TNF, IL6, or IL1B. Genes involved in degradation of the extracellular matrix (e.g. MMP2, MMP9), hypoxia regulated genes $(H I F 1 A)$ and proangiogenic genes (GPNMB, VEGFA, IGF1) were also part of the ercDC profile. Of note was the robust expression of VSIG4 in ercDC_ccRCC. Surface protein expression of VSIG4, GPNMB, NRP1 and CD9 was validated by FACS (Figure $2 \mathrm{H})$.

Collectively, these results indicated that ercDC_cCRCC combine features of various macrophage polarization states. High mRNA expression of MMP2 and MMP9 confirmed our previously described protein data and the angiogenic signature identified supports the hypothesis that ercDCs may help promote tumor growth (Figel et al., 2011). The strong expression of factors linked to the induction of T cell tolerance, MAF (Cao et al., 2005) and VSIG4 (Vogt et al., 2006), support a potential immunoregulatory role for ercDCs in ccRCC tissue. VSIG4 was strongly expressed on the protein level, while other well described markers of immunoinhibition (PD-L1/B7-H1 and PD-L2/B7- 
DC) (Latchman et al., 2001; Saunders et al., 2005) were only marginally expressed.

TIM-3 showed weak expression and B7-H3 was strongly expressed (Figure S1E).

\section{ErcDCs have a gene expression signature similar to inflammatory macrophages from ascites of ovarian cancer with characteristics of immune tolerance}

Renal tubulo-interstitial DCs are described to act as sentinels maintaining homeostasis and protecting the renal tubuli from immune-induced injury through tolerogenic mechanisms (Scholz et al., 2008). To investigate whether ercDCs arising in the tubulointerstitial milieu of $\mathrm{ccRCC}$ similarly exhibit tolerizing features that might confer tumor immune protection we conducted a global analysis across published transcriptomes. The reference data comprised human myeloid cell types from blood and various non-lymphoid tissues, including myeloid subsets originating from tissues with described tolerogenic milieus (detailed information in supplemental procedures and table S2B). In brief, the subsets tested were $\mathrm{CD} 11 \mathrm{c}^{\mathrm{HI}}$ and $\mathrm{CD} 11 \mathrm{c}^{\mathrm{LO}}$ decidual macrophages (Houser et al., 2011), three DC subtypes from the lamina propria distinguished by their expression of CD103 and Sirpa (CD103 ${ }^{+}$Sirpa $^{+}$DCs, CD103Sirpa $^{+}$DCs, CD103 ${ }^{+}$Sirpa ${ }^{-}$DCs) (Watchmaker et al., 2014), alveolar macrophages from non-smokers, smokers and COPD or asthma patients (Shaykhiev et al., 2009; Woodruff et al., 2005), human TAMs from gastrointestinal stromal tumors (GIST) (Cavnar et al., 2013), two myeloid cell types from the ascites of ovarian cancer patients (inflammatory macrophages (infMФ_ascOvCa) and inflammatory DCs (infDC_ascOvCa)) (Segura et al., 2013), and CD141+ DCs from peripheral blood (Lindstedt et al., 2005). We supplemented the published data sets with in-house expression profiles of PBMC-derived monocytes, slanDCs and CD1C ${ }^{+}$DCs.

Hierarchical clustering revealed similiarity of ercDC_ccRCC with MФ_ccRCC, infMФ_ascOvCa and infDC_ascOvCa (Figure 3A). CD11 $\mathrm{c}^{\mathrm{LO}}$ MФ_decidua, 
TAM_GIST, CD103+Sirpa ${ }^{+} D C \_g u t$ and MФ_asthma_avlung formed a separate subgroup. Blood-derived cell types, together with CD103+Sirpa- DC_gut and CD103Sirpa $^{+}$DC_gut clustered distinct from all other cell types. CD141+ DC_blood more closely resembled CD103+Sirpa-DC_gut, confirming the similarity in characteristics previously described, including expression of markers associated with crosspresentation (Watchmaker et al., 2014). Principle component analysis (PCA) (Figure 3B) provided further evidence that ercDC_ccRCC are most similar to MФ_ccRCC and infMФ_ascOvCa, and are clearly different from blood-derived cells.

To assess if expression states of specific genes can distinguish ercDC_ccRCC from other myeloid subtypes, we used the nearest shrunken centroids method (NSCM) (Tibshirani et al., 2002), a supervised machine learning approach suited to define subsets of genes that best characterize specific cellular states. Feature selection on the classifier that we trained to predict ercDC_ccRCC revealed 61 marker genes as predictive for ercDC (Figure S2C). Hierarchical clustering of all myeloid cell types based on expression of the 61 marker genes (Figure 4A) showed that ercDC_ccRCC clearly separated from the other human cell types analyzed with the exceptions of infMФ_ascOvCa and CD11 $c^{\mathrm{LO}} \mathrm{M} \Phi$ _decidua. Despite originating from the same tissue, MФ_ccRCC were positioned in a different cluster together with infDC_ascOvCa, and were thus not classified as ercDC_ccRCC. The tumor-associated macrophages from GIST (TAM_GIST), which are described as an antitumoral M1-like TAM subtype, did not show similarities with the ercDC_ccRCC profile. Blood-derived myeloid cells,

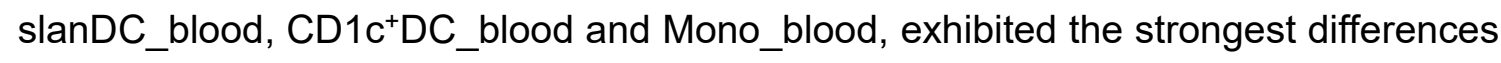
to the ercDC_ccRCC profile, with an almost inverse expression of many of the marker genes.

Thirty-nine of the 61 marker genes were upregulated while 22 were downregulated in ercDC_ccRCC as compared to the other cell types (Figure 4A, table S7). As expected, 
CD209/DC-SIGN, which was used to distinguish ercDC_ccRCC from MФ_ccRCC in FACS, was present in the marker gene list and showed increased expression (Figure 4A). The macrophage core gene SEPP1, the M2-associated gene MAF and the M1associated gene CXCL9 were present in the ercDC_ccRCC marker gene list, as well as genes associated with immunoinhibitory and proangiogenic functions, such as GPNMB and NRP1.

Given the strong similarity of ercDCs and infMФ_ascOvCa we definded a list of differentially expressed genes (DEGs) between these two cell types and the other myeloid cell types. The genes with significantly different expression (FDR <0.05) in ercDC\&infMФ_ascOvCa as compared to the other samples (control group) are shown in Figure 4B and were designated ercDC_ccRCC DEGs. They include 788 genes, with 431 showing upregulation and 357 downregulation (tables S8, S9). The DEG list includes 54 of the 61 marker genes (89\%), which are shown in bold letters with yellow background in the volcano plot (Figure 4B). Most of the marker genes showing the strongest and most significant expression differences were upregulated DEGs, e.g. CD209/DC-SIGN, FOLR2, GPNMB and SEPP1. The upregulated DEGs included genes associated with antiinflammatory function and macrophage recruitment. These include CD204/MSR1, MERTK, CD163, CCL2, CCL8 and CCL18 (Figure 4B). Downregulated DEGs included ercDC_ccRCC marker genes (FAM65B, FGR, CFP) and DC-associated genes, including BCL11A and FLT3.

Superposition of M1- and M2-associated genes (table S3, S4) on the list of informative ercDC_ccRCC genes again illustrated the mosaic expression of M2- and M1associated genes by this myeloid subtype (Figure 4C). Among the most significantly upregulated genes were the M2-associated genes, CD209/DC-SIGN, SLCO2B1, SLC38A6, SEPP1, CCL18, MAF, MS4A4A and IGF1, but also the M1-associated genes IL2RA and CXCL9. These M1/M2-associated genes also belonged to the 
ercDC_ccRCC marker genes. Overall, $2.6 \%$ of the M1-associated genes and $12.9 \%$ of the M2-associated genes were among the ercDC_ccRCC marker genes. Moreover, $23.7 \%$ of M1-associated genes and $45.2 \%$ of M2-associated genes were part of the ercDC_ccRCC DEGs. GSEA analysis provided evidence of significance for the difference in expression of the M2-gene set $(p=0.02)$ and enrichment of the M1-gene set $(p=0.09)$ in the ercDC_ccRCC\&infMФ_ascOvCa. This suggests that ercDC_ccRCC may represent a hybrid myeloid subtype with mosaic features of M2and M1-polarization.

To identify enriched biological processes associated with the DEGs, GO term analysis was employed. The results showed "response to wounding" and "inflammatory response" as the most significantly scored categories (Figure 5A). These effector processes correspond with the described inflammatory milieu in RCC (Dvorak, 1986; Fox et al., 2013) and the well recognized role for renal macrophages in normal tissue homeostasis and wound healing (Nelson et al., 2012; Kawakami et al., 2013). The third most enriched category was "defense response", underscoring a potential bactericidal activity of ercDC_ccRCC, which agrees with the general expression of M1-associated genes.

The results of InnateDB and GSEA (based on the data bases "KEGG", Reactome" and "Biocarta") analyses overlapped with GO term analysis (table S10). Most of the enriched pathways identified are associated with the complement system, lipid metabolism and modulation of the extracellular matrix. Deregulated lipid metabolism has been described to promote the development and progression of RCC (Drabkin and Gemmill, 2010).

GeneMANIA network analysis was conducted to better characterize the functional network of ercDC_ccRCC marker genes, and to identifiy functionally related genes (Figure 5B). The related genes identified (grey) included many macrophage- 
associated genes, particularly those of M2-macrophages and their immunoinhibitory function, CD163, CD206/MRC1, CD14 and VSIG4 (yellow circles in Figure 5B).

\section{ErcDCs are distinct from blood-derived monocytes from RCC patients}

Recently it was reported that blood-derived monocytes from RCC patients display a tumor-promoting transcription profile (Chittezhath et al., 2014). Inflammatory blood monocytes can act as precursors for TAMs (Caso et al., 2010; Movahedi et al., 2010; Qian et al., 2011). Hierarchical clustering and PCA evaluation including the RCCmonocyte transcriptome (designated as Mono_RCC_blood) clearly separated the Mono_RCC_blood from the ercDC_ccRCC and clustered the Mono_RCC_blood with CD11 ${ }^{\mathrm{HI}}$ MФ_decidua and CD103-Sirpa+ DC_gut (Figure 6A, B).

Tissue-specific gene expression can obscure cell type-specific profiles. However, even after exclusion of blood-specific genes ("tissue preferential expressed gene list (blood genes)" (Mele et al., 2015)) Mono_RCC_blood remained clearly distinct from ercDC_ccRCC and retained their similarity with the CD11 $\mathrm{C}^{\mathrm{HI}} \mathrm{M}_{\mathrm{M}} \Phi_{\text {decidua and }} \mathrm{CD} 10^{-}$ Sirpa+DC_gut (Figure S3A, B). Clustering based on the ercDC_ccRCC marker genes positioned the Mono_RCC_blood distant to ercDC_ccRCC in a subcluster together with $\mathrm{CD} 11 \mathrm{C}^{\mathrm{HI}} \mathrm{M} \Phi$ _decidua (Figure 6C), similar to the hierarchical cluster analysis on all informative genes (Figure 6A). Identical clustering was observed after exclusion of blood-specific genes (Figure S3C).

A comparison of the expression of M1- and M2-associated genes between RCCmonocytes and ercDCs revealed that Mono_RCC_blood resembled ercDC_ccRCC in their expression of M1-associated genes, but were more similar to CD1C ${ }^{+}$DC_blood in their expression of M2-associated genes, while ercDC_ccRCC clustered with M2macrophages (Figure 6D). Removal of the blood-specific genes again did not change this profile (Figure S3D). Mono_RCC_blood are thought to derive their protumoral 
activity through an interleukin-1 receptor (IL-1R)-dependent mechanism. Comparing the IL-1R pathway activation by GSEA showed that the corresponding genes were less expressed $(p=0.0001)$ in ercDC_ccRCC as compared to Mono_RCC_blood (Figure $6 \mathrm{E})$.

\section{Expression of ercDC marker genes in ccRCC tissue is predictive of patient}

\section{survival}

We have previously shown that CD209+ cell numbers were higher in advanced ccRCC tumors with poor prognostic tumor stage (Figel et al., 2011). The transcriptome analysis described here further supports a role of ercDCs in tumor promotion and immunoinhibiton. As shown in Figure 7, an ercDC score based on the expression of the ercDC marker genes was found to be associated with cancer-specific survival (CSS). The association was significant for the large Cancer Genome Atlas (TCGA) cohort of 442 ccRCC samples $(H R=1.8, p=3.0 E-02)$ and an independent cohort of 28 ccRCCs (Rostock cohort) (table S12) $(\mathrm{HR}=4.8, \mathrm{p}=8.8 \mathrm{E}-03)$. In both cohorts, patients with high ercDC score showed decreased CSS as compared to patients with low ercDC score. In addition, the ercDC score correlated with tumor grade (Figure 7B, D) in both cCRCC cohorts.

\section{The ercDC_ccRCC polarization profile reveales distinct tissue imprints}

Recent reports have suggested that the phenotype and function of tissue-resident macrophages is robustly influenced by factors present in the tissue micromilieus (Gordon et al., 2014; Davies et al., 2013; Das et al., 2015). To investigate the potential influence of the ccRCC milieu on the gene expression profile of ercDC_ccRCC, predefined stimulus-specific human gene sets were used that are known to be induced by distinct macrophage activation signals (Xue et al., 2014). We correlated the module 
eigengenes (ME) of the stimulus-specific gene sets (so-called modules) with the gene expression profile of ercDC_ccRCC, various human myeloid cell types from nonlymphoid tissues, including Mono_RCC_blood, and in vitro-generated M1- and M2macrophages (Figure 7E, table S11). The ercDC_ccRCC clustered closest with infMФ_ascOvCa, confirming the strong relationship seen previously using the NSCM. Macrophages from the ccRCC tissues (MФ_ccRCC), M2-MФ_in vitro and infDC_ascOvCA clustered within the same group (no. 4). All cell types from this group also showed largely negative correlations with modules in cluster A, linked to signals associated with the cytokine IL-4. On the other hand, they showed predominantly strong positive associations with modules in cluster B. The modules of cluster B were generally linked to signals from glucocorticoids (GC), palmitic acid (PA), prostaglandin E2 (PGE $)$ or a combination of TNF, PGE 2 and P3C (Pam3CysSerLys4, TLR2-ligand, TPP) (in orange letters). Other modules in cluster B are linked to M1-polarizing stimuli (IFN-y, TNF) (in brown letters); accordingly, a very strong positive correlation was found with in vitro-polarized M1-macrophages (M1_MФ_in vitro) and a negative correlation with M2_MФ_in vitro. ErcDC_ccRCC also correlated negatively or showed no correlation, while infMФ_ascOvCA, infDC_ascOvCA and MФ_ccRCC showed some positive correlation. Overall, Mono_RCC_blood showed much weaker polarization than ercDC_ccRCC, illustrating that the two cell types have different tissue origins.

In summary, the data suggest that the characteristic transcriptional profile of ercDCs may be induced in part by GC, PGE 2 , PA and TPP, and absence of IL-4. This is consistent with described characteristics of the RCC tissue milieu, featuring GC (Huynh et al., 2015), PGE 2 (Wu et al., 2011) and TNF (Gogusev et al., 1993; Chuang et al., 2008) and lacking IL-4 (Hosse, 2009). PA has been described as playing a role in kidney fibrosis (Kang et al., 2015). The emerging imprinting stimuli are also 
consistent with the marker profile determined using NSCM and the upregulated DEGs. $\mathrm{PGE}_{2,}$ for example, is described to induce the transcription of MSR1/CD204 (DomingoGonzalez et al., 2013), a gene also strongly expressed in ercDC_ccRCC. Moreover, $\mathrm{PGE}_{2}$ and GC are known inducers of CD163 (Schaer et al., 2002; Zhang et al., 2014), another marker strongly upregulated in ercDC_ccRCC. In addition, GC regulates the expression of MERTK as well as C1QB, CCL8, VSIG4 and FCN1, all of which belong to the ercDC_ccRCC marker genes or DEGs.

\section{VSIG4 on ercDCs and contacts with FOXO1-positive T cells in situ}

Based on the observation that a high ercDC score is associated with poor patient survival, we then examined the transcriptional profile of ercDCs for genes linked to immune evasion and tumor progression in more detail. In particular, VSIG4 appeared among the immunoregulatory and proangiogenic/invasive features and emerged as a gene functionally related to the marker genes in the GeneMANIA network analysis. ErcDC_ccRCC strongly expressed VSIG4 at the RNA (Figure 8A) and protein level at the cell surface (Figure 8B, C). Multiparameter immunofluorescence histology of ccRCC tissues showed that the majority (85-98\%) of CD209 ${ }^{+}$cells coexpressed VSIG4. Tumors from advanced tumor stages harbored more CD209+VSIG4+ cells than those of earlier tumor stages (abs. median number 45.7, range 21-75; vs 35, range 3158). We have previously reported that ercDCs are in tight contact with T cells in ccRCC tissue (Figel et al., 2011). We had additionally observed that the T cells from ccRCC tissue are functionally inactive with a molecular signature of anergy, including suppression of ERK and AKT pathways and high expression of cell cycle inhibitor p27kip (Prinz et al., 2012). We therefore asked whether the cell cycle arrest in T cells could be caused through T-cell contact with ercDCs expressing VSIG4, thereby inducing $\mathrm{p} 27^{\mathrm{kip}}$. As $\mathrm{p} 27^{\mathrm{kip}}$ is a downstream target of FOXO1, which is a transcription 
factor that is negatively regulated by AKT, we evaluated the expression of VSIG4 and FOXO1 in ercDC-T cell contacts in situ using immunohistology. We observed that many $\mathrm{T}$ cells $(12-41 \%)$ in ccRCC tissue stained positive for FOXO1. FOXO1 was localized to the nucleus indicating an active protein (Figure 8D), which is consistent with suppressed AKT pathway in ccRCC-resident T cells. FOXO1 was preferentially expressed in T cells that interacted with VSIG4+ ercDCs compared to T cells not in contact with VSIG4 ${ }^{+}$ercDCs (Figure 8E). In late stage tumors (T105, T114, T118), the majority of the $\mathrm{FOXO}^{+} \mathrm{T}$ cells were found in contact with $\mathrm{VSIG} 4^{+}$cells contrasting early stage tumor (T90). 


\section{Discussion}

Renal cell carcinoma (RCC) has long been recognized as an immune-responsive tumor with a well documented sensitivity to T-cell attack dating from the 1970s (Kim et al., 2003; Motzer, 2003; Vogelzang and Stadler, 1998). Yet, clinical studies using to immune checkpoint blockade therapy that releases $T$ cells from tumor-induced inhibition by targeting the co-inhibitory protein CTLA-4 or the PD-1/L1 axis (Sharma and Allison, 2015) document that RCC patients do not benefit substantially more compared to patients with tumors previously considered to be non-immunogenic (Sunshine and Taube, 2015). This observation indicates the existence of additional layers of tumor-mediated immunosuppression beyond $\mathrm{T}$ cell checkpoints that hamper antitumor immune responses and need to be addressed to improve efficacy of cancer immunotherapy.

We have previously shown that $\mathrm{T}$ cells infiltrating human $\mathrm{cCRCC}$ display a signature of cell cycle inhibition with suppressed ERK and AKT pathway activation (Prinz et al., 2012). Many of the T cells seen in ccRCC tissue are tightly engaged with CD209/CD14 double-positive myeloid cells (Figel et al., 2011). Here we provide evidence for tumorpromoting qualities as well as immunoinhibitory characteristics of ercDCs that may translate to T-cell dysfunction. A high ercDC score was found to be strongly associated with poor patient survival, showing prognostic value and suggesting that ercDCs may be targets for therapeutic intervention. Targeting ercDCs, particularly in situations, where tumors have high ercDC content, may expand the range of patients that can be effectively treated by immunotherapy.

The ercDC transcriptome revealed details regarding their functional polarization and positioning within the MPS. The transcriptomic data suggest that ercDCs belong to the macrophage lineage as they strongly expressed human macrophage-associated core genes, transcription factors and growth factor receptors. Notably, they show combined 
features of M1-macrophages and M2-macrophages as well as gene signatures associated with wound healing and tissue remodeling, immunoregulation, and bactericidal effector activities. These observations were supported by GO term, InnateDB and GSEA analyses, showing "response to wounding", "inflammatory response" and "defense response" as the most significantly enriched categories. Furthermore, pathways associated with the complement system, lipid metabolism and modulation of the extracellular matrix were highly enriched. These features are strongly associated with the inflammatory milieu in RCC tissue (Dvorak, 1986; Fox et al., 2013) and the general role of macrophages in tissue homeostasis and wound healing in kidney biology (Kawakami et al., 2013; Nelson et al., 2012). An altered lipid metabolism is a characteristic of ccRCC (Pinthus et al., 2011; Rezende et al., 1999) as is the accumulation of immune complexes with accompanying complement activation, which is also seen in many inflammatory renal diseases (Bagavant and Fu, 2009; NikolicPaterson and Atkins, 2001). The identification of these complex features for a myeloid cell subtype enriched in RCC confirms and extends previous studies that have reported diametrically polarized macrophages in RCC tissue (Kovaleva et al., 2016).

A comparative analysis across published transcriptome databases of human myeloid cell types from blood and non-lymphoid tissues using the NSCM method showed an association with an inflammatory macrophage subtype from the ascites of ovarian cancer (infMФ_ascOvCa) as closest relative to ercDC_ccRCC. Described features for ovarian TAMs include the expression of M2-markers (CD14, CD206, CD11b, CD204), select M1-markers (CD86, TNF) (Allavena et al., 2010; Bellora et al., 2014; Colvin, 2014; Kawamura et al., 2009; Merogi et al., 1997; Segura et al., 2013), and expression of the immunosuppressive and antiinflammatory chemokine CCL18. These expression patterns parallel those seen in ercDC_ccRCC. Interestingly, cytokines shown to induce the ercDC phenotype in vitro, IL-6, CXCL8/IL-8 and VEGF (Figel et al., 2011), are also 
present in ovarian ascites (Giuntoli et al., 2009; Lane et al., 2011; Reinartz et al., 2014). Thus, the relatedness of ercDC_ccRCC and infM $\Phi$ _ascOvCa may be explained in part by their exposure to a related tissue milieu. In addition, $\mathrm{PGE}_{2}, \mathrm{GC}, \mathrm{TNF}$, palmitic acid (PA) and TLR2-ligands were identified as ercDC_ccRCC polarizing factors. $\mathrm{PGE}_{2}$ is an important factor in RCC biology (Wu et al., 2011). The enrichment in $\mathrm{PGE}_{2}$ and $\mathrm{GC}$ is linked to the expression of CD163 (Schaer et al., 2002; Zhang et al., 2014) in ercDCs. In addition, GC has been described to induce MerTK (Zizzo et al., 2012) and VSIG4 (Gorgani et al., 2011), and as such, may be an underlying mechanism for the expression of those markers in ercDCs.

By hierarchical clustering of the transcriptome data of ercDC_ccRCC and other myeloid cell types, ercDCs clearly separated from all blood-derived myeloid cells, including blood-derived monocytes from RCC patients, which were described to potentially represent precursors of RCC-TAMs (Caso et al., 2010; Chittezhath et al., 2014; Movahedi et al., 2010; Qian et al., 2011). Hierarchical clustering and classification based on the ercDC_ccRCC marker genes similarly identified the Mono_blood_RCC as blood-derived myeloid cells with a transcriptome distinct from tissue-derived cell types including ercDCs_ccRCC, even after elimination of bloodspecific genes. Based on the results we conclude that ercDCs represent a cell type that is resident to non-lymphoid tissues displaying a distinct polarization state imparted by tissue-specific signals. Indeed, we were not able to detect $C D 209^{+} C D 14^{+} C D 163^{+}$ myeloid cells among peripheral blood mononuclear cells by FACS (data not shown) and the ercDCs score was strongly decreased in samples of acute myeloid leukemia of the TCGA collection compared to ccRCCs (not shown).

Several genes within the ercDC marker profile suggest T-cell inhibitory effector activity. While PD-L1, PD-L2 and TIM-3 were only marginally expressed by ercDCs, prominent cell surface expression was found for the immunoinhibitory proteins VSIG4 (He et al., 
2008; Ikarashi et al., 2013; Tanaka et al., 2012; Vogt et al., 2006) and GPNMB (Glycoprotein NMB, DC-associated transmembrane protein (DC-HIL), osteoactivin) (Chung et al., 2007; Ripoll et al., 2007). These markers represent novel targets to potentially reverse ercDC-mediated T cell inhibition. VSIG4 may be involved in the Tcell dysfunction associated with overexpressed $\mathrm{p} 27^{\mathrm{kip}}$, previously described by our group (Prinz et al., 2012). T cells in contact with VSIG4+ ercDCs preferentially expressed FOX01, a positive regulator of the tolerance-inducing p27 ${ }^{\mathrm{kip}}$. NRP1 (Miyauchi et al., 2016) and CSF-1R are additional potential targets expressed by ercDCs where therapeutic reagents are currently in phase I studies (Link: https://clinicaltrials.gov/ct2/show/NCT02452424).

The results presented identify new potential mechanisms for immunoregulation in ccRCC malignancy involving direct communication between ercDCs and T cells. The next generation of studies will help determine whether the functional attributes suggested by the ercDC transcriptome are found in one single cell, or if they are represented by the assembly of multiple cell types with different functions. Single cell transcriptomics and multiplex mass cytometry may help clarify this issue. Our analysis using polychromatic flow cytometry indicates that the cells co-expressing the ercDC index markers, CD209 and CD14, largely also co-express the M1-marker CD64 and two M2-markers, CD204 and MerTK. This provides a first indication that the ercDCs may be a unique cell type on the single cell level with mixed macrophage polarization. Recently, macrophages from RCC tissues were analyzed by highly multiplex mass cytometry (Chevrier et al., 2017). Unfortunately, the analysis did not include the CD209 marker nor other relevant ercDC signature genes identified here, thus precluding a comparative analysis of our ercDCs to 17 TAM phenotypes identified in the mass cytometry study. Mass cytometry is a powerful approach, but as a front-line analysis tool it is disadvantaged by its bias towards previously characterized markers. By 
contrast, the transcriptomic and bioinformatics-based analysis described here allowed the identification of a novel series of proteins, including VSIG4 and GPNMB, or NRP1 and CSF1R, that likely contribute to T-cell suppression and tumor immune escape in ccRCC. In addition to providing new markers for mass cytometry studies, these proteins also represent potential targets for immunotherapy, and combined with checkpoint blockade, or other therapeutic strategies, may improve treatment outcome, especially for tumors with a high ercDC score. 


\section{Experimental procedures}

\section{Tissues, cells, cell culture}

Tissue and blood collection were approved by the local ethics commission of the LMU München, and patients/donors consented to the donation. Tissue samples of histologically diagnosed clear cell renal cell carcinoma (ccRCC) $(n=15)$ were obtained from untreated patients who underwent surgery at the Urologische Klinik Dr. Castringius Planegg (Munich, Germany). Patient characteristics are shown in table S1. Fresh postoperative material was used to prepare cell suspensions and cryosections (Figel et al., 2011). Peripheral blood mononuclear cells (PBMCs) from healthy donors (HD) were used to isolate monocytes (using CD14 ${ }^{+}$microbeads, Miltenyi Biotec) and to sort CD1 $\mathrm{C}^{+} \mathrm{DC}$ and slanDCs using FACS (Aria IIlu, BD). Before cell sorting, PBMCs were depleted of $\mathrm{B}$ and $\mathrm{NK}$ cells via $\mathrm{CD} 19^{+}$and $\mathrm{CD}^{+} 6^{+}$microbeads according to the manufacturer's protocol (Miltenyi Biotec). M1- and M2- macrophages were generated from blood monocytes in vitro, as described (Martinez et al., 2006). Briefly, monocytes were cultured in 6-well plates (Nunc) in serum-free medium ( $5 \times 10^{6} / 4 \mathrm{ml}$ of AIM-V) supplemented with M-CSF (50 ng/ml; R\&D Systems) over 7 days followed by $18 \mathrm{~h}$ treatment with IFN-y (20 ng/ml; R\&D Systems) plus LPS (100 ng/ml; Sigma-Aldrich) for M1-polarization, or IL-4 (20 ng/ml; PromoKine) for M2-polarization.

\section{Cell sorting}

ErcDCs and macrophages were sorted from ccRCC tissue-cell-suspensions stained with CD45-PeCy7, CD11c-APC, CD3-PB, CD209-PE (all BD Biosciences), CD14PerCPCy5.5 (eBioscience) and LIVE/DEAD® Fixable Near-IR Dead Cell Stain Kit (Thermo Fisher Scientific). Sorting gates were set on CD209+CD14+ ${ }^{+}$cells (ercDCs) and CD209-CD14+ cells (macrophages), among pre-gated CD45+, live, single CD11C ${ }^{+}$ CD3- cells. CD1C ${ }^{+}$DC and slanDCs were sorted from B- and NK-depleted PBMCs of 
healthy donors (HD) using anti-CD11C-PE, anti-CD3-PB (all BD Biosciences), antiCD56-APC (Beckman Coulter), anti-CD19-PB (Dako), anti-CD1c-PeCy7 (Biolegend), anti-slan-FITC (Miltenyi Biotec) and LIVE/DEAD® Fixable Near-IR Dead Cell Stain Kit. Gating strategy and instrument parameters are in supplemental Figure S2. Gates were set very strictly, not covering the whole population, to avoid contamination with other cell populations. Cell population purity varied between $98 \%-100 \%$. Cells were directly sorted into $250 \mu$ l of RLT lysis buffer with ß-mercaptoethanol (RNeasy Micro Kit, Qiagen) using FACSAria Illu (BD Biosciences), then homogenized (QIAshredder, Qiagen) and stored at $-80^{\circ} \mathrm{C}$. Details about sorted cell types, number of biological replicates are listed in table S2).

\section{Polychromatic flow cytometry}

$1-5 \times 10^{5}$ cells were incubated with antibodies for $30 \mathrm{~min} / 4^{\circ} \mathrm{C}$ and LIVE/DEAD $®$ Fixable Near-IR or Blue fluorescent Dead Cell Stain Kit, washed, optionally incubated with secondary antibodies and acquired at LSRII (BD Biosciences). In tissue-cellsuspensions, CD45 was used to identify leukocytes after exclusion of dead cells and doublets. Myeloid cells were selected based on CD11c (pan-myeloid marker in human) with exclusion of $\mathrm{CD}^{+} \mathrm{T}$ cells. Within $\mathrm{CD} 11 \mathrm{c}^{+}$cells, ercDCs and macrophages were distinguished as CD209/CD14 double-positive cells (ercDCs) and CD14 singlepositive cells (macrophages). For M1/M2-macrophages, live cells where selected and doublets excluded before marker analysis. Antibodies are listed in table S13.

\section{Immunofluorescence histology and confocal microscopy}

Cryosections were fixed with $4 \%$ paraformaldehyde (PFA, Merck) and stained with primary and fluorescent-labeled secondary antibodies as described (Figel et al., 2011). The antibody combinations were: goat-anti-human VSIG4 (IgG, R\&D) and mouse-anti- 
human CD209/DC-SIGN (mouse IgG2a, SantaCruz Biotechnology) followed by secondary antibodies donkey anti-goat-A488 (ThermoScientific) and rat anti-mouseIgG-Cy5 or donkey anti-mouse-IgG-A647 (both Jackson ImmuneResearch); goat-antihuman VSIG4, rabbit-anti-human FOXO1 (Cell Signaling) and mouse-anti-human CD3 (UCHT1, BD) followed by secondary antibodies donkey anti-goat-A488, donkey antirabbit-RRX (Jackson ImmuneResearch) and donkey anti-mouse-A647 or rat antimouse IgG-Cy5. Slides were mounted with ProLong® Gold Antifade (ThermoFischer Scientific). Fluorescence images were captured with a laser scanning microscope TCS SP5 Leica Microsystems, Wetzlar, Germany) with settings as described (Figel et al., 2011).

\section{RNA isolation, microarray hybridization}

RNA was prepared using RNeasy micro kit (Qiagen). RNA quantity and quality was assessed using a Nanodrop 1000 Spectrometer (Peqlab) and Agilent 2100 Bioanalyzer. From in vitro-generated cells (M1-, M2-macrophages) and CD14 monocytes, three replica pools (each consisting of RNA from 5 different HD) were generated. RNA from flow-sorted cells was not pooled (table S2). RNA (30 ng of each replica pool; 0.5-15 ng of sorted cells) was subsequently amplified and converted into cDNA by a linear amplification method using WT-Ovation PicoSL System in combination with the Encore ${ }^{\circledR}$ Biotin Module (both from NuGen, San Carlos, US). cDNA was hybridized to Affymetrix GeneChip® Human Gene 1.0 ST Arrays.

\section{Microarray data preprocessing and probe set filtering}

Raw intensity data were processed with R/Bioconductor (Bioconductor.org). If not stated otherwise, functions were called with default parameters. We calculated normalized expression values for each study group (in-house generated and external 
data sets, see table S2 and supplemental experimental procedure) independently using Robust Multichip Average (RMA, library 'oligo') preprocessing including background correction and quantile normalization. Technical control probe sets as well as probe sets whose values did not vary between arrays (variance $=0$ ) were excluded from all further analyses. Many-probe-sets-to-one-gene relationships were resolved by keeping only one probe set with highest variance for each gene. Further analyses included only informative genes, which we defined as group with the $50 \%$ most variable expression within the respective study group.

\section{Combining microarray studies}

For comparing transcript levels across studies, gene expression values were merged based on annotated gene entrez ids and study batch effects were removed using the COMBAT method (Johnson et al., 2007) (library "inSilicoMerging"). We included all samples of a study in the merge and selected samples of interest afterwards.

\section{Hierarchical clustering and heatmaps}

Gene and samplewise hierarchical clustering of expression profiles used euclidean distances and complete agglomeration method. Heatmaps represent color-coded genewise standardized expression levels (mean $=0$, standard deviation $=1$; $z$-score).

\section{Marker genes}

Nearest shrunken centroid classifiers (Tibshirani et al., 2002) were constructed with the function "pamr.train" and cross-validated with the function "pamr.cv" (library "pamr") (Figure S2C) on informative genes of compared data sets. The classification threshold (1.88) was set such that the false positive classification rate was smaller than $20 \%$ and a preferably small number of genes was obtained. 
GeneMANIA network analysis (Warde-Farley et al., 2010) was conducted based on the ercDC_ccRCC marker genes with default parameters (data as of May 2014).

Differentially expressed genes (DEGs) between ercDC_ccRCC\&infM $\Phi$ _ascOvCa and control group (all other samples/groups listed in table S2) were identified by a linear model using empirical Bayes moderated t-tests (R package "limma") and BenjaminiHochberg correction for multiple testing. DEGs were defined by an adjusted $p$-value < 0.05 .

For Gene ontology enrichment (GO) analysis, hypergeometric p-values for enrichment or depletion of differentially expressed genes in GO categories of the group "biological process" were calculated with the function "hyperGTest", Bioconductor package "GOstats". Informative genes served as background and p-value threshold was set to 0.001

Enrichment of differentially expressed genes within InnateDB (Breuer et al., 2013, Lynn et al., 2010, Lynn et al., 2008) signaling pathway gene sets (from "KEGG“, "BioCarta“, "Reactome", "NetPath", "INOH" und "PID") was defined with default settings at InnateDB.com (data as of May 2014). Informative genes served as background.

GSEA analysis was performed with GSEA 1.0 R-script of the Broad institute with default parameters (Subramanian et al., 2005) (http://www.broad.mit.edu/gsea/).

For describing gene-expression-module-to-cell-type relationships, we first calculated median expression values per gene for each cell type. Eigengene values for gene expression modules as described (Xue et al., 2014) were calculated with the function "moduleEigengens" from the R-package "WGCNA" (Langfelder and Horvath, 2008). Eigengenes were correlated to cell types using Pearson's method.

ercDC score in the Cancer Genome Atlas (TCGA) and the validation (Rostock) cohort 
Transcriptome profiling data ("HTSeq - FPKM-UQ") of the TCGA ccRCC cohorts (KIRC, LAML) were downloaded from the Genomic Data Commons Portal (https://gdcportal.nci.nih.gov/) on December 9, 2016 and December 15, 2016, respectively. Clinical data were obtained from the same platform on October 10, 2016 and November 3, 2016, respectively. To be used in this study, TCGA samples had to meet the following criteria: Patients with neoadjuvant therapies ("history_of_neoadjuvant_treatment") were excluded. Moreover, only subjects with available survival data were considered (overall survival, OS, for the LAML cohort; cancer-specific survival (CSS) as defined in (Buttner et al., 2015) for the ccRCC cohort). Follow-up time was required to be greater than 0 . Missclassified patients (Chen et al., 2016; Buttner et al., 2015) revealed by cluster analysis and/or by reevaluation of tissue histology were also discarded from the TCGA ccRCC cohort. Characteristics of the final TCGA ccRCC cohort $(n=442$ patients) are summarized in table S12.

The validation cohort included a collection of selected G1 and G3 ccRCC tissues ( $\mathrm{n}=$ 14 each) and corresponding normal kidney tissue $(n=14)$ microarray data ("Rostock cohort”, Array GeneChip HG U133 Plus 2.0, Affymetrix) (Maruschke et al., 2011; Maruschke et al., 2014). Clinical data and recent follow up are found in table S12.

An ercDC score was established by gene expression deconvolution. Expression values of the 61 marker genes in the ercDC arrays were first collapsed by taking the median. FPKM-UQ expression values in the TCGA ccRCC cohort were logtransformed $(\log 2(x+1))$. Subsequently, using the marker gene set, a simple linear regression model was fit with "expression in ercDC cell type" as predictor and "expression in sample" as regressor. Finally, the slope of the linear model constituted the ercDC score of a sample. Conditional inference trees from R-package partykit_1.11 (Hothorn et al., 2006b, Hothorn and Zeileis, 2015) 
http://jmlr.org/papers/v16/hothorn15a.html were used to identify groups with significantly varying CSS curves in both the TCGA CCRCC cohort and the validation cohort. The $p$-value criterion of the conditional inference tree method was weakened (0.1) and the minimum group size was set to 10 . Kaplan-Meier curves and corresponding log-rank tests using R-package survival_2.40-1 (Therneau and Grambsch, 2000) were applied for survival analysis. Further, R-package coin_1.1-3 (Hothorn et al., 2006a) was used to perform a linear trend test between ercDC score and tumor grade.

\section{Public access to raw data of data sets analyzed in this paper}

Our data sets of human ercDCs and macrophages from ccRCC tissue, myeloid cells from PBMCs and in vitro-generated M1- and M2-macrophages are accessible via super series GSE108312.

\section{Author contributions}

Conception and design: D.B., E.N., P.J.N.; acquistion of data: D.B., E.K.; Microarray hybridization: M.I., J.B.; Bioinformatics: T.S., F.B., E.S. S.W., M.S., D.B.; data interpretation and manuscript writing: D.B., E.N., P.J.N., T.S., F.B., E.S., S.W., M.S.; Study supervision: E.N.. All authors approved the manuscript.

\section{Acknowledgements}

We acknowledge A. Buchner and M. Maruschke for providing the Rostock microarray information, M. Schmitz, R. Wehner, R. Oberneder for providing tissues/tissue suspensions. We thank J. Ellwart, J. Mysliwietz, A. Brandl, A. Bettenbrock, C. Jäckel 
and B. Mosetter for technical help. We thank The Cancer Genome Atlas initiative (http://cancergenome.nih.gov/), all tissue donors and investigators who contributed to the acquisition and analyses of the samples used in this study. This work was supported by Robert Bosch Foundation (Stuttgart, Germany), ICEPHA Graduate School Tuebingen-Stuttgart, Deutsche Krebshilfe, the Helmholtz Alliance 'Aging and Metabolic Programming, AMPro' and DGF SFB-TR36. The authors declare no conflicts of interests.

\section{Supplemental information}

Supplemental information includes 3 figures and 13 tables and can be found with this article online at super series GSE108312. 


\section{References}

Allavena, P., Chieppa, M., Bianchi, G., Solinas, G., Fabbri, M., Laskarin, G. \& Mantovani, A. (2010). Engagement of the mannose receptor by tumoral mucins activates an immune suppressive phenotype in human tumor-associated macrophages. Clin Dev Immunol 2010, 547179.

Bagavant, H. \& Fu, S. M. (2009). Pathogenesis of kidney disease in systemic lupus erythematosus. Curr Opin Rheumatol 21, 489-94.

Bellora, F., et al. (2014). TLR activation of tumor-associated macrophages from ovarian cancer patients triggers cytolytic activity of NK cells. Eur J Immunol 44, 181422.

Breuer, K., et al. (2013). InnateDB: systems biology of innate immunity and beyond-recent updates and continuing curation. Nucleic Acids Res 41, D1228-33.

Buttner, F., et al. (2015). Survival Prediction of Clear Cell Renal Cell Carcinoma Based on Gene Expression Similarity to the Proximal Tubule of the Nephron. Eur Urol 68, 1016-20.

Cao, S., Liu, J., Song, L. \& Ma, X. (2005). The protooncogene c-Maf is an essential transcription factor for IL-10 gene expression in macrophages. J Immunol 174, 348492.

Carlo, M. I., Voss, M. H. \& Motzer, R. J. (2016). Checkpoint inhibitors and other novel immunotherapies for advanced renal cell carcinoma. Nat Rev Urol 13, 420-31.

Caso, R., Silvera, R., Carrio, R., Iragavarapu-Charyulu, V., Gonzalez-Perez, R. R. \& Torroella-Kouri, M. (2010). Blood monocytes from mammary tumor-bearing mice: early targets of tumor-induced immune suppression? Int J Oncol 37, 891-900.

Cavnar, M. J., et al. (2013). KIT oncogene inhibition drives intratumoral macrophage M2 polarization. J Exp Med 210, 2873-86.

Chen, F., et al. (2016). Multilevel Genomics-Based Taxonomy of Renal Cell Carcinoma. Cell Rep 14, 2476-89.

Chevrier, S., et al. (2017). An Immune Atlas of Clear Cell Renal Cell Carcinoma. Cell $169,736-749$ e18.

Chittezhath, M., et al. (2014). Molecular profiling reveals a tumor-promoting phenotype of monocytes and macrophages in human cancer progression. Immunity 41, 815-29.

Chuang, M. J., Sun, K. H., Tang, S. J., Deng, M. W., Wu, Y. H., Sung, J. S., Cha, T. L. \& Sun, G. H. (2008). Tumor-derived tumor necrosis factor-alpha promotes progression and epithelial-mesenchymal transition in renal cell carcinoma cells. Cancer Sci 99, 905-13.

Chung, J. S., Sato, K., Dougherty, li, Cruz, P. D., Jr. \& Ariizumi, K. (2007). DC-HIL is a negative regulator of T lymphocyte activation. Blood 109, 4320-7. 
Colvin, E. K. (2014). Tumor-associated macrophages contribute to tumor progression in ovarian cancer. Front Oncol 4, 137.

Das, A., Sinha, M., Datta, S., Abas, M., Chaffee, S., Sen, C. K. \& Roy, S. (2015). Monocyte and macrophage plasticity in tissue repair and regeneration. Am J Pathol $185,2596-606$.

Davies, L. C., Jenkins, S. J., Allen, J. E. \& Taylor, P. R. (2013). Tissue-resident macrophages. Nat Immunol 14, 986-95.

Domingo-Gonzalez, R., Katz, S., Serezani, C. H., Moore, T. A., Levine, A. M. \& Moore, B. B. (2013). Prostaglandin E2-induced changes in alveolar macrophage scavenger receptor profiles differentially alter phagocytosis of Pseudomonas aeruginosa and Staphylococcus aureus post-bone marrow transplant. J Immunol 190, 5809-17.

Drabkin, H. A. \& Gemmill, R. M. (2010). Obesity, cholesterol, and clear-cell renal cell carcinoma (RCC). Adv Cancer Res 107, 39-56.

Dvorak, H. F. (1986). Tumors: wounds that do not heal. Similarities between tumor stroma generation and wound healing. N Engl J Med 315, 1650-9.

Engel, D. R., Krause, T. A., Snelgrove, S. L., Thiebes, S., Hickey, M. J., Boor, P., Kitching, A. R. \& Kurts, C. (2015). CX3CR1 reduces kidney fibrosis by inhibiting local proliferation of profibrotic macrophages. J Immunol 194, 1628-38.

Figel, A. M., et al. (2011). Human renal cell carcinoma induces a dendritic cell subset that uses T-cell crosstalk for tumor-permissive milieu alterations. Am J Pathol 179, 436-51.

Fox, P., Hudson, M., Brown, C., Lord, S., Gebski, V., De Souza, P. \& Lee, C. K. (2013). Markers of systemic inflammation predict survival in patients with advanced renal cell cancer. Br J Cancer 109, 147-53.

Gautier, E. L., et al. (2012). Gene-expression profiles and transcriptional regulatory pathways that underlie the identity and diversity of mouse tissue macrophages. Nat Immunol 13, 1118-28.

Giraldo, N. A., et al. (2015). Orchestration and Prognostic Significance of Immune Checkpoints in the Microenvironment of Primary and Metastatic Renal Cell Cancer. Clin. Cancer Res. 21, 3031-40.

Giraldo, N. A., et al. (2017). Tumor-Infiltrating and Peripheral Blood T-cell Immunophenotypes Predict Early Relapse in Localized Clear Cell Renal Cell Carcinoma. Clin. Cancer Res. 23, 4416-4428.

Giuntoli, R. L., 2nd, Webb, T. J., Zoso, A., Rogers, O., Diaz-Montes, T. P., Bristow, R. E. \& Oelke, M. (2009). Ovarian cancer-associated ascites demonstrates altered immune environment: implications for antitumor immunity. Anticancer Res 29, 287584. 
Gogusev, J., Augusti, M., Chretien, Y. \& Droz, D. (1993). Interleukin-6 and TNF alpha production in human renal cell carcinoma. Kidney Int 44, 585-92.

Gordon, S., Pluddemann, A. \& Martinez Estrada, F. (2014). Macrophage heterogeneity in tissues: phenotypic diversity and functions. Immunol Rev 262, 36-55.

Gordon, S. \& Taylor, P. R. (2005). Monocyte and macrophage heterogeneity. Nat Rev Immunol 5, 953-64.

Gorgani, N. N., Thathaisong, U., Mukaro, V. R., Poungpair, O., Tirimacco, A., Hii, C. S. \& Ferrante, A. (2011). Regulation of CRIg expression and phagocytosis in human macrophages by arachidonate, dexamethasone, and cytokines. Am J Pathol 179, 1310-8.

Guilliams, M., Ginhoux, F., Jakubzick, C., Naik, S. H., Onai, N., Schraml, B. U., Segura, E., Tussiwand, R. \& Yona, S. (2014). Dendritic cells, monocytes and macrophages: a unified nomenclature based on ontogeny. Nat Rev Immunol 14, 571-8.

Gustafsson, C., Mjosberg, J., Matussek, A., Geffers, R., Matthiesen, L., Berg, G., Sharma, S., Buer, J. \& Ernerudh, J. (2008). Gene expression profiling of human decidual macrophages: evidence for immunosuppressive phenotype. PLoS One 3, e2078.

Hansel, A., Gunther, C., Ingwersen, J., Starke, J., Schmitz, M., Bachmann, M., Meurer, M., Rieber, E. P. \& Schakel, K. (2011). Human slan (6-sulfo LacNAc) dendritic cells are inflammatory dermal dendritic cells in psoriasis and drive strong TH17/TH1 T-cell responses. J Allergy Clin Immunol 127, 787-94 e1-9.

He, J. Q., Wiesmann, C. \& Van Lookeren Campagne, M. (2008). A role of macrophage complement receptor CRIg in immune clearance and inflammation. Mol Immunol 45, 4041-7.

Hochheiser, K., et al. (2013). Exclusive CX3CR1 dependence of kidney DCs impacts glomerulonephritis progression. J Clin Invest 123, 4242-54.

Hochheiser, K. \& Kurts, C. (2015). Selective Dependence of Kidney Dendritic Cells on CX3CR1--Implications for Glomerulonephritis Therapy. Adv Exp Med Biol 850, 55-71.

Hosse, J. 2009. Die molekulare Quantifizierung von NK-Zellen im klarzelligen Nierenzellkarzinom und die Bedeutung von CCL5 in NK-Zellen und für die Phagozytose. Ludwig-Maximilians-University Munich.

Hothorn, T., Hornik, K., Van De Wiel, M. A. \& Zeileis, A. (2006a). A Lego System for Conditional Inference. The American Statistician 60, 257-263.

Hothorn, T., Hornik, K. \& Zeileis, A. (2006b). Unbiased Recursive Partitioning: A Conditional Inference Framework. Journal of Computational and Graphical Statistics $15,651-674$.

Hothorn, T. \& Zeileis, A. (2015). partykit: A Modular Toolkit for Recursive Partytioning in R. Journal of Machine Learning Research 16, 3905-3909. 
Houser, B. L., Tilburgs, T., Hill, J., Nicotra, M. L. \& Strominger, J. L. (2011). Two unique human decidual macrophage populations. J Immunol 186, 2633-42.

Hume, D. A. \& Freeman, T. C. (2014). Transcriptomic analysis of mononuclear phagocyte differentiation and activation. Immunol Rev 262, 74-84.

Huynh, T. P., et al. (2015). Glucocorticoids suppress renal cell carcinoma progression by enhancing Na,K-ATPase beta-1 subunit expression. PLoS One 10, e0122442.

Ikarashi, M., Nakashima, H., Kinoshita, M., Sato, A., Nakashima, M., Miyazaki, H., Nishiyama, K., Yamamoto, J. \& Seki, S. (2013). Distinct development and functions of resident and recruited liver Kupffer cells/macrophages. J Leukoc Biol 94, 1325-36.

Jantzer, P. \& Schendel, D. J. (1998). Human renal cell carcinoma antigen-specific CTLs: antigen-driven selection and long-term persistence in vivo. Cancer Res 58, 3078-86.

Johnson, W. E., Li, C. \& Rabinovic, A. (2007). Adjusting batch effects in microarray expression data using empirical Bayes methods. Biostatistics 8, 118-27.

Kang, H. M., et al. (2015). Defective fatty acid oxidation in renal tubular epithelial cells has a key role in kidney fibrosis development. Nat Med 21, 37-46.

Kawakami, T., et al. (2013). Resident renal mononuclear phagocytes comprise five discrete populations with distinct phenotypes and functions. J Immunol 191, 3358-72.

Kawamura, K., Komohara, Y., Takaishi, K., Katabuchi, H. \& Takeya, M. (2009). Detection of M2 macrophages and colony-stimulating factor 1 expression in serous and mucinous ovarian epithelial tumors. Pathol Int 59, 300-5.

Kim, H. L., Belldegrun, A. S. \& Figlin, R. A. (2003). Immune gene therapy for kidney cancer: the search for a magic trigger. Mol Ther 7, 153-4.

Kovaleva, O. V., Samoilova, D. V., Shitova, M. S. \& Gratchev, A. (2016). Tumor Associated Macrophages in Kidney Cancer. Anal Cell Pathol (Amst) 2016, 9307549.

Lane, D., Matte, I., Rancourt, C. \& Piche, A. (2011). Prognostic significance of IL-6 and IL-8 ascites levels in ovarian cancer patients. BMC Cancer 11, 210.

Langfelder, P. \& Horvath, S. (2008). WGCNA: an R package for weighted correlation network analysis. BMC Bioinformatics 9, 559.

Latchman, Y., et al. (2001). PD-L2 is a second ligand for PD-1 and inhibits T cell activation. Nat Immunol 2, 261-8.

Leisegang, M., Turqueti-Neves, A., Engels, B., Blankenstein, T., Schendel, D. J., Uckert, W. \& Noessner, E. (2010). T-cell receptor gene-modified T cells with shared renal cell carcinoma specificity for adoptive T-cell therapy. Clin Cancer Res 16, 233343. 
Lindstedt, M., Lundberg, K. \& Borrebaeck, C. A. (2005). Gene family clustering identifies functionally associated subsets of human in vivo blood and tonsillar dendritic cells. J Immunol 175, 4839-46.

Lynn, D. J., et al. (2010). Curating the innate immunity interactome. BMC Syst Biol 4, 117.

Lynn, D. J., et al. (2008). InnateDB: facilitating systems-level analyses of the mammalian innate immune response. Mol Syst Biol 4, 218.

Mantovani, A. and Sica, A. (2010). Macrophages, innate immunity and cancer: balance, tolerance, and diversity. Curr Opin Immunol 22, 231-7.

Mantovani, A., Sica, A., Sozzani, S., Allavena, P., Vecchi, A. \& Locati, M. (2004). The chemokine system in diverse forms of macrophage activation and polarization. Trends Immunol 25, 677-86.

Martinez, F. O., Gordon, S., Locati, M. \& Mantovani, A. (2006). Transcriptional profiling of the human monocyte-to-macrophage differentiation and polarization: new molecules and patterns of gene expression. J Immunol 177, 7303-11.

Martinez, F. O., Helming, L. \& Gordon, S. (2009). Alternative activation of macrophages: an immunologic functional perspective. Annu Rev Immunol 27, 451-83.

Martinez, F. O., Sica, A., Mantovani, A. \& Locati, M. (2008). Macrophage activation and polarization. Front Biosci 13, 453-61.

Maruschke, M., Hakenberg, O. W., Koczan, D., Zimmermann, W., Stief, C. G. \& Buchner, A. (2014). Expression profiling of metastatic renal cell carcinoma using gene set enrichment analysis. Int J Urol 21, 46-51.

Maruschke, M., Reuter, D., Koczan, D., Hakenberg, O. W. \& Thiesen, H. J. (2011). Gene expression analysis in clear cell renal cell carcinoma using gene set enrichment analysis for biostatistical management. BJU Int 108, E29-35.

Mele, M., et al. (2015). Human genomics. The human transcriptome across tissues and individuals. Science 348, 660-5.

Merogi, A. J., Marrogi, A. J., Ramesh, R., Robinson, W. R., Fermin, C. D. \& Freeman, S. M. (1997). Tumor-host interaction: analysis of cytokines, growth factors, and tumorinfiltrating lymphocytes in ovarian carcinomas. Hum Pathol 28, 321-31.

Miller, J. C., et al. (2012). Deciphering the transcriptional network of the dendritic cell lineage. Nat Immunol 13, 888-99.

Miyauchi, J. T., Chen, D., Choi, M., Nissen, J. C., Shroyer, K. R., Djordevic, S., Zachary, I. C., Selwood, D. \& Tsirka, S. E. (2016). Ablation of Neuropilin 1 from gliomaassociated microglia and macrophages slows tumor progression. Oncotarget 7, 980114. 
Morris, D. L., Singer, K. \& Lumeng, C. N. (2011). Adipose tissue macrophages: phenotypic plasticity and diversity in lean and obese states. Curr Opin Clin Nutr Metab Care 14, 341-6.

Mosser, D. M. (2003). The many faces of macrophage activation. J Leukoc Biol 73, 209-12.

Mosser, D. M. \& Edwards, J. P. (2008). Exploring the full spectrum of macrophage activation. Nat Rev Immunol 8, 958-69.

Motzer, R. J. (2003). Renal cell carcinoma: a priority malignancy for development and study of novel therapies. J Clin Oncol 21, 1193-4.

Motzer, R. J., et al. (2015). Nivolumab versus Everolimus in Advanced Renal-Cell Carcinoma. N Engl J Med 373, 1803-13.

Movahedi, K., et al. (2010). Different tumor microenvironments contain functionally distinct subsets of macrophages derived from Ly6C(high) monocytes. Cancer Res 70, 5728-39.

Murray, P. J., et al. (2014). Macrophage activation and polarization: nomenclature and experimental guidelines. Immunity 41, 14-20.

Nelson, P. J., Rees, A. J., Griffin, M. D., Hughes, J., Kurts, C. \& Duffield, J. (2012). The renal mononuclear phagocytic system. J Am Soc Nephrol 23, 194-203.

Nikolic-Paterson, D. J. \& Atkins, R. C. (2001). The role of macrophages in glomerulonephritis. Nephrol Dial Transplant 16 Suppl 5, 3-7.

Nizzoli, G., et al. (2013). Human CD1c+ dendritic cells secrete high levels of IL-12 and potently prime cytotoxic T-cell responses. Blood 122, 932-42.

Pinthus, J. H., Whelan, K. F., Gallino, D., Lu, J. P. \& Rothschild, N. (2011). Metabolic features of clear-cell renal cell carcinoma: mechanisms and clinical implications. Can Urol Assoc J 5, 274-82.

Pollard, J. W. (2009). Trophic macrophages in development and disease. Nat Rev Immunol 9, 259-70.

Prinz, P. U., Mendler, A. N., Masouris, I., Durner, L., Oberneder, R. \& Noessner, E. (2012). High DGK-alpha and disabled MAPK pathways cause dysfunction of human tumor-infiltrating CD8+ $\mathrm{T}$ cells that is reversible by pharmacologic intervention. $\mathrm{J}$ Immunol 188, 5990-6000.

Qian, B. Z., Li, J., Zhang, H., Kitamura, T., Zhang, J., Campion, L. R., Kaiser, E. A., Snyder, L. A. \& Pollard, J. W. (2011). CCL2 recruits inflammatory monocytes to facilitate breast-tumour metastasis. Nature 475, 222-5.

Reinartz, S., et al. (2014). Mixed-polarization phenotype of ascites-associated macrophages in human ovarian carcinoma: correlation of CD163 expression, cytokine levels and early relapse. Int J Cancer 134, 32-42. 
Rezende, R. B., Drachenberg, C. B., Kumar, D., Blanchaert, R., Ord, R. A., loffe, O. B. \& Papadimitriou, J. C. (1999). Differential diagnosis between monomorphic clear cell adenocarcinoma of salivary glands and renal (clear) cell carcinoma. Am J Surg Pathol 23, $1532-8$.

Ripoll, V. M., Irvine, K. M., Ravasi, T., Sweet, M. J. \& Hume, D. A. (2007). Gpnmb is induced in macrophages by IFN-gamma and lipopolysaccharide and acts as a feedback regulator of proinflammatory responses. J Immunol 178, 6557-66.

Saunders, P. A., Hendrycks, V. R., Lidinsky, W. A. \& Woods, M. L. (2005). PD-L2:PD1 involvement in $\mathrm{T}$ cell proliferation, cytokine production, and integrin-mediated adhesion. Eur J Immunol 35, 3561-9.

Schaer, D. J., Boretti, F. S., Schoedon, G. \& Schaffner, A. (2002). Induction of the CD163-dependent haemoglobin uptake by macrophages as a novel anti-inflammatory action of glucocorticoids. Br J Haematol 119, 239-43.

Scholz, J., Lukacs-Kornek, V., Engel, D. R., Specht, S., Kiss, E., Eitner, F., Floege, J., Groene, H. J. \& Kurts, C. (2008). Renal dendritic cells stimulate IL-10 production and attenuate nephrotoxic nephritis. J Am Soc Nephrol 19, 527-37.

Segura, E., Touzot, M., Bohineust, A., Cappuccio, A., Chiocchia, G., Hosmalin, A., Dalod, M., Soumelis, V. \& Amigorena, S. (2013). Human inflammatory dendritic cells induce Th17 cell differentiation. Immunity 38, 336-48.

Sharma, P. \& Allison, J. P. (2015). Immune checkpoint targeting in cancer therapy: toward combination strategies with curative potential. Cell 161, 205-14.

Shaykhiev, R., Krause, A., Salit, J., Strulovici-Barel, Y., Harvey, B. G., O'connor, T. P. \& Crystal, R. G. (2009). Smoking-dependent reprogramming of alveolar macrophage polarization: implication for pathogenesis of chronic obstructive pulmonary disease. $\mathrm{J}$ Immunol 183, 2867-83.

Sica, A. \& Mantovani, A. (2012). Macrophage plasticity and polarization: in vivo veritas. J Clin Invest 122, 787-95.

Soos, T. J., Sims, T. N., Barisoni, L., Lin, K., Littman, D. R., Dustin, M. L. \& Nelson, P. J. (2006). CX3CR1+ interstitial dendritic cells form a contiguous network throughout the entire kidney. Kidney Int 70, 591-6.

Subramanian, A., et al. (2005). Gene set enrichment analysis: a knowledge-based approach for interpreting genome-wide expression profiles. Proc Natl Acad Sci U S A $102,15545-50$.

Sunshine, J. \& Taube, J. M. (2015). PD-1/PD-L1 inhibitors. Curr Opin Pharmacol 23, 32-8.

Tanaka, M., Nagai, T., Usami, M., Hasui, K., Takao, S. \& Matsuyama, T. (2012). Phenotypic and functional profiles of CRIg (Z39lg)-expressing macrophages in the large intestine. Innate Immun 18, 258-67. 
Tarique, A. A., Logan, J., Thomas, E., Holt, P. G., Sly, P. D. \& Fantino, E. (2015). Phenotypic, functional, and plasticity features of classical and alternatively activated human macrophages. Am J Respir Cell Mol Biol 53, 676-88.

Therneau, T. M. \& Grambsch, P. M. (2000). Modeling Survival Data: Extending the Cox Model. Springer, New York

Tibshirani, R., Hastie, T., Narasimhan, B. \& Chu, G. (2002). Diagnosis of multiple cancer types by shrunken centroids of gene expression. Proc Natl Acad Sci U S A 99, 6567-72.

Van Kooyk, Y., Unger, W. W., Fehres, C. M., Kalay, H. \& Garcia-Vallejo, J. J. (2013). Glycan-based DC-SIGN targeting vaccines to enhance antigen cross-presentation. Mol Immunol 55, 143-5.

Vogelzang, N. J. \& Stadler, W. M. (1998). Kidney cancer. Lancet 352, 1691-6.

Vogt, L., et al. (2006). VSIG4, a B7 family-related protein, is a negative regulator of T cell activation. J Clin Invest 116, 2817-26.

Wang, T., et al. (2012). Melanoma-derived conditioned media efficiently induce the differentiation of monocytes to macrophages that display a highly invasive gene signature. Pigment Cell Melanoma Res 25, 493-505.

Warde-Farley, D., et al. (2010). The GeneMANIA prediction server: biological network integration for gene prioritization and predicting gene function. Nucleic Acids Res 38, W214-20.

Watchmaker, P. B., et al. (2014). Comparative transcriptional and functional profiling defines conserved programs of intestinal DC differentiation in humans and mice. Nat Immunol 15, 98-108.

Woodruff, P. G., Koth, L. L., Yang, Y. H., Rodriguez, M. W., Favoreto, S., Dolganov, G. M., Paquet, A. C. \& Erle, D. J. (2005). A distinctive alveolar macrophage activation state induced by cigarette smoking. Am J Respir Crit Care Med 172, 1383-92.

Wu, J., Zhang, Y., Frilot, N., Kim, J. I., Kim, W. J. \& Daaka, Y. (2011). Prostaglandin E2 regulates renal cell carcinoma invasion through the EP4 receptor-Rap GTPase signal transduction pathway. J Biol Chem 286, 33954-62.

Xue, J., et al. (2014). Transcriptome-based network analysis reveals a spectrum model of human macrophage activation. Immunity 40, 274-88.

Zhang, M., et al. (2014). Liver myofibroblasts up-regulate monocyte CD163 expression via PGE2 during hepatitis B induced liver failure. J Transl Med 12, 60.

Zhang, X. \& Mosser, D. M. (2008). Macrophage activation by endogenous danger signals. J Pathol 214, 161-78. 
Zizzo, G., Hilliard, B. A., Monestier, M. \& Cohen, P. L. (2012). Efficient clearance of early apoptotic cells by human macrophages requires M2c polarization and MerTK induction. J Immunol 189, 3508-20. 


\section{Figure legends}

Figure 1: Human macrophage and DC core genes in ercDC_ccRCC. (A) Clustered heatmaps depicting relative gene expression levels of human macrophage and DC core genes comparing ercDC, in vitro-generated M1- and M2-macrophages, and CD1 $\mathrm{C}^{+} \mathrm{DC}$ from blood. Genes whose expression was validated on protein level $(B, C)$ are set apart. (B), (C) Protein expression by flow cytometry. In vitro-generated M1- and M2-macrophages, PBMCs and ccRCC tissue cell suspensions were stained with marker combinations and gated on ercDCs (CD209 ${ }^{+} \mathrm{CD} 14^{+}$cells among $\mathrm{CD}^{+} 5^{+}$live

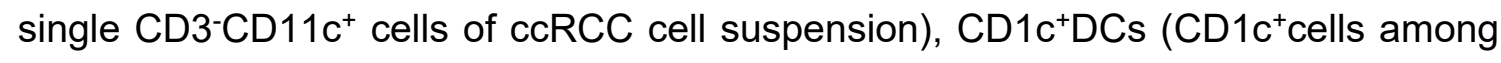
live single $C D 3^{-} C D 11 c^{+}$of PBMCs) and on M1- and M2-macrophage population among live single cells. Depicted are representative histograms of human macrophage markers CD64A, CD14, MerTK, CD32A (B) as well as DC markers ANPEP/CD13 and FLT3 (C) from at least 6 different patient tissues or PBMCs. Black line histogram: specific staining, gray filled histogram: control staining. Numbers indicate the controlcorrected median fluorescence intensity.

Figure 2: DC- and macrophage-associated genes and genes associated with angiogenesis and invasion in ercDCs. (A) Gene expression of macrophageassociated transcription factors and growth factor receptor CSF1R. Bars: median of each group, symbols correspond to individual array replicates of a cell type. Histogram depicts surface expression of CSF1R on ercDCs of $c \mathrm{RCC}$ tissue cell suspensions by flow cytometry (B) Linear expression of genes for DC-associated transcription factors and growth factor receptor FLT3. (C) Clustered heatmaps depicts relative expression levels of genes associated with M1- and M2-macrophage polarization. Surface protein levels of indicated markers validated by flow cytometry. See table S3 and S4 for M1and M2-genes. (D) Dot plot of polychromatic flow cytometry demonstrates co- 
expression of M1- (CD64A) and M2-macrophage markers (CD204/MSR1 and MerTK) on ercDCs in ccRCC tissue cell suspensions $(n=4)$. Overlay of MerTK ${ }^{+}$cells on gated

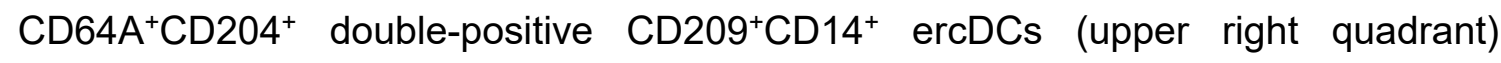
depicted in pink. (E) Linear expression of chemokines associated with host defense activity of macrophages. M1-macrophages are the positive reference. Bars: median of each group; symbols correspond to individual array replicates of a cell type. (F), (G) Clustered heatmap of relative expression levels of genes associated with wound healing and tissue remodeling $(F)$ and immunoregulation $(G)$. References are M2macrophages and CD11 $\mathrm{C}^{\mathrm{LO}}$ macrophages from decidua (CD11C ${ }^{\mathrm{LO}} \mathrm{M \Phi}$ _decidua). $(\mathrm{H})$ Clustered heatmap depicting relative expression levels of genes associated with angiogenesis and invasion. Positive reference: in vitro-generated M2-macrophages; negative control: CD1c $c^{+}$Cs from blood. Surface expression of indicated proteins on ercDCs by flow cytometry of ccRCC tissue-cell suspensions and gating on ercDCs

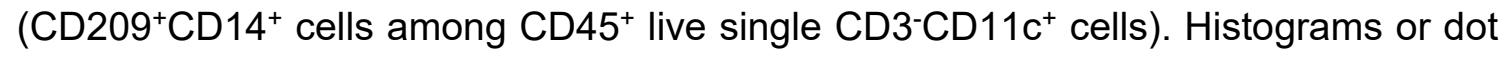
plots are representative of at least $n=6$ ) different tissue suspensions. Black line histogram: specific stainings, filled grey histogram: control stainings. Numbers indicate the control-corrected median fluorescence intensity.

Figure 3: Transcriptome relationship of ercDC_ccRCC and myeloid cell types from blood and non-lymphatic healthy and pathological tissues. (A) Hierachical clustering of indicated cell types based on median expression values of replicate samples considering only the top $50 \%$ of genes with highest variation across cell types (6107 "informative" genes). (B) PCA on same data set as described in (A). Shown are the first two PC that describe $28 \%$ and $15 \%$ of the variance, respectively. 
Figure 4: Marker gene profile and DEGs of ercDC_ccRCC. (A) Clustered heatmap of relative expression levels of 61 marker genes calculated with NSCM (see also Figure S2) comparing expression levels of ercDCs_ccRCC with those of all other cell types listed in tables S2 (control group). Dashed box highlights the gene CD209/DC-SIGN, which was used to distinguish ercDC_ccRCC from MФ_ccRCC in FACS. (B) Volcano plot depicting differences in gene expression across informative genes of ercDC_ccRCC\&infMФ_ascOvCa $(n=11)$ and control group $(n=177)$. DEGs are red symbols (adjusted $p<0.05$ ). Symbols with assigned gene name have a grey edge. ErcDC_ccRCC marker genes are in bold and highlighted in yellow; green: genes discussed in the text.

(C) Volcano plot depicting M1- and M2-macrophage-associated genes (orange and green); (grey): informative genes resulting from the comparison of ercDC_ccRCC\&infMФ_ascOvca and the control group. DEGs among informative genes are located above the dotted horizontal line $(p<0.05)$. The table shows the number and percentage of ercDC_ccRCC\&infMФ_ascOvCa DEGs and marker genes (bold) within the informative M1- and M2-macrophage-associated genes.

Figure 5: GO categories and functional network of ercDC_ccRCC. (A) Upregulated DEGs were enriched in 19 of the top 20 categories, downregulated DEGs were only enriched in one category ("Translational initiation") of the top 20 significantly enriched terms. Significance of GO category enrichment is depicted as -log10 (p-value). (B) Functional associations of ercDC_ccRCC marker genes (black) and their computed related genes (grey) by GeneMANIA network analysis. Yellow circles highlight related genes mentioned in the text. Associations were defined based on different criteria (coloured lines, see Figure). 


\section{Figure 6: Transcriptome comparison of ercDC_ccRCC with published blood} monocytes from RCC patients. All figures are based on expression levels with bloodspecific genes not excluded. See Figure S3 for results after exclusion of blood-specific genes. (A) Hierachical clustering and (B) PCA of gene expression profiles. Analyses are based on informative genes for which the median expression value of replicates for each cell type was calculated. (C) Clustered heatmap of ercDC_ccRCC marker genes with blood monocytes of RCC patients (Mono_RCC_blood). (D) Clustered heatmaps showing relative expression levels of genes associated with M1- and M2macrophages in Mono_RCC_blood and ercDC_ccRCC. (E) Enrichment plot for 73 IL1R pathway genes defined by merging MSIGDB sets "BIOCARTA_IL1R_PATHWAY", "REACTOME_IL1_SIGNALING", "PID_IL1_PATHWAY" and “INTERLEUKIN_1_SECRETION”. Genes indicated by vertical bars are spread along the x-axis based on t-statistic comparing ercDC_ccRCC and Mono_RCC_blood cells. Enrichment worm on top shows the relative enrichment of genes in each part of the plot.

Figure 7: Association of ercDC_ccRCC marker gene profile with cancer-specific survival and specific polarization stimuli $(A)(C)$ Kaplan-Meier estimates of cancerspecific survival (CSS) of ccRCC tumors from the TCGA cohort $(n=442)$ and the Rostock cohort $(n=28)$ predicted by the ercDC score. Partitioning into ercDClow and ercDC $^{\text {high }}$ using conditional inference trees with endpoint CSS. (B) (D) Box plots of ercDC score values of TCGA ccRCC tumors $(n=439$, no grading was available for 3 samples of TCGA cohort, see table 12) and normal kidney tissues ( $n=65)$ or the Rostock tumors ( $n=14$ for grade G1, G3, and normal kidney tissues) ( $p$-value by linear trend test). (E) Heatmap of Pearson correlation coefficients calculated between module eigengenes (ME) and myeloid cell types. Cell types were clustered based on 
correlations with stimuli-associated eigengen modules. Depicted are the numbered modules with up to two significantly positive correlated stimuli for each module. Stimuli dominating in module group A are highlighted green, in module group B in orange and brown. Stimuli negatively correlating with the modules as well as the correlation rules for stimuli and modules are listed in table S11.

Figure 8: VSIG4 on ercDCs and contacts with FOX01-positive T cells. (A) Linear expression of VSIG4. Bars are the median of each group; symbols correspond to individual array replicates of a cell type. CD1c ${ }^{+} D C \_b l o o d$ is the negative reference, M2-MФ_in vitro the positive reference. (B) Validation of VSIG4 protein on ercDCs in ccRCC tissue cell suspension by flow cytometry. ercDCs are gated as CD209+CD14 ${ }^{+}$ cells among $C D 45^{+}$live single $C D 3^{-} C D 11 c^{+}$cells in ccRCC cell suspension, $C D 1 c^{+} D C s$ are gated within PBMCs as $\mathrm{CD}_{1} \mathrm{c}^{+}$cells among live single CD3-CD11 ${ }^{+}$cells. Control staining: grey filled histogram. Numbers depict the difference in median fluorescence intensity (MFI) between specific antibody and control. Shown is a representative example of $n=4$ different $c c R C C$ tissue cell suspensions. (C) Confocal images of ccRCC tissues stained with CD209 (red), VSIG4 (green) and DAPI (blue). Original magnification, x400. Cells coexpressing CD209 and VSIG4 are yellow. Images are merged fluorescent channels and maximal projection of 6-9 z-planes (z-step size $=0.7$ $\mu \mathrm{m})$. Left image: late stage ccRCC (RCC114, T3cG2), right image: early stage ccRCC (RCC90, T1aG2), (D). Confocal image of an ccRCC tissue area (RCC118) depicting a $\mathrm{T}$ cell $\left(\mathrm{CD}^{+}\right.$, cyan) expressing $\mathrm{FOXO1}$ (red) in contact with $\mathrm{VSIG}^{+}$cell (original magnification, x400, zoom 5). Histograms document fluorescence intensities of a single z-plane along indicated line. (E) Scatter plot showing the ratio of the \% $\mathrm{FOXO}^{+}$ T cells among $\mathrm{T}$ cells that are in contact with $\mathrm{VSIG}^{+}$cells to the $\%$ of $\mathrm{FOXO}^{+} \mathrm{T}$ cells among $\mathrm{T}$ cells that are not in contact with $\mathrm{VSIG} 4^{+}$cells. Values above $>1$ indicate that 
bioRxiv preprint doi: https://doi.org/10.1101/2020.01.20.912865; this version posted January 27, 2020. The copyright holder for this preprint (which was not certified by peer review) is the author/funder. All rights reserved. No reuse allowed without permission.

the majority of $\mathrm{CD}^{+} \mathrm{FOXO}^{+}$cells is in contact with VSIG4 ${ }^{+}$cells. ${ }^{* * *}=p<0.001$, Mann Whitney test comparing across all 4 ccRCC tissues. 


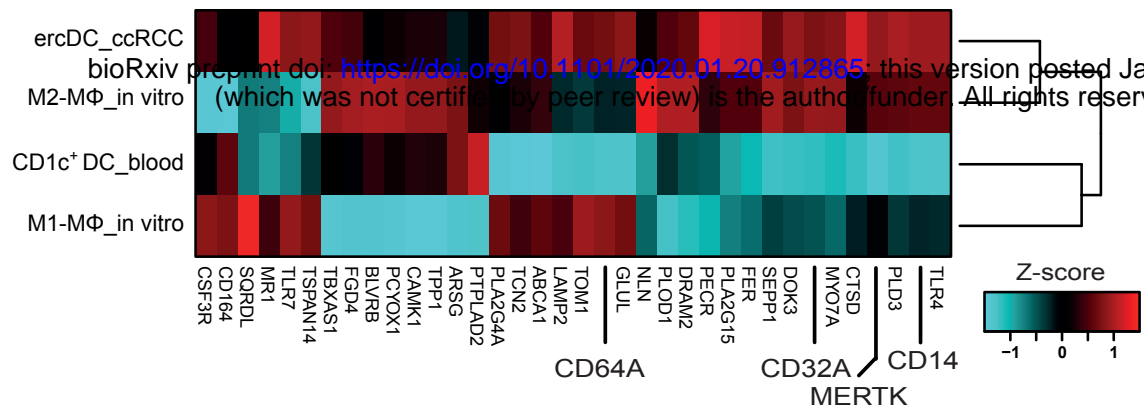

rsion pested January 27, 2020. The copyright holder for this preprint All rights reserved. No reuse allowed without permission.

DC core genes

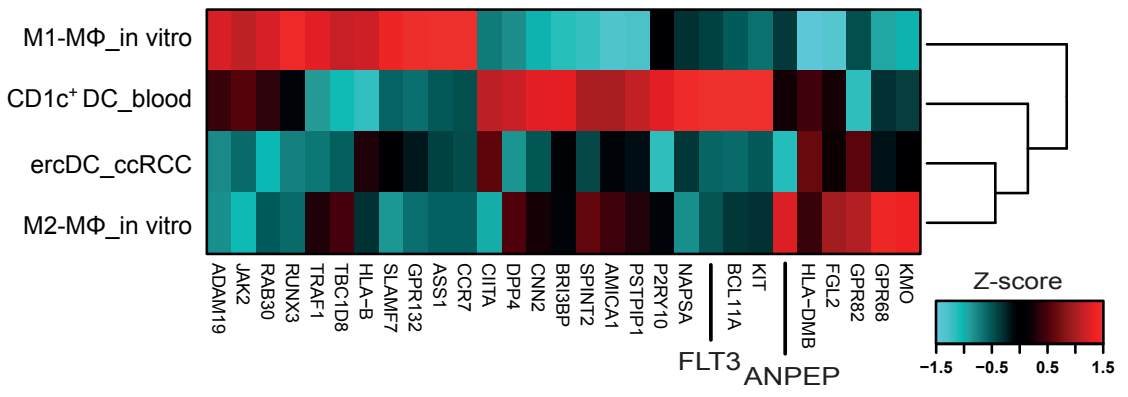

B

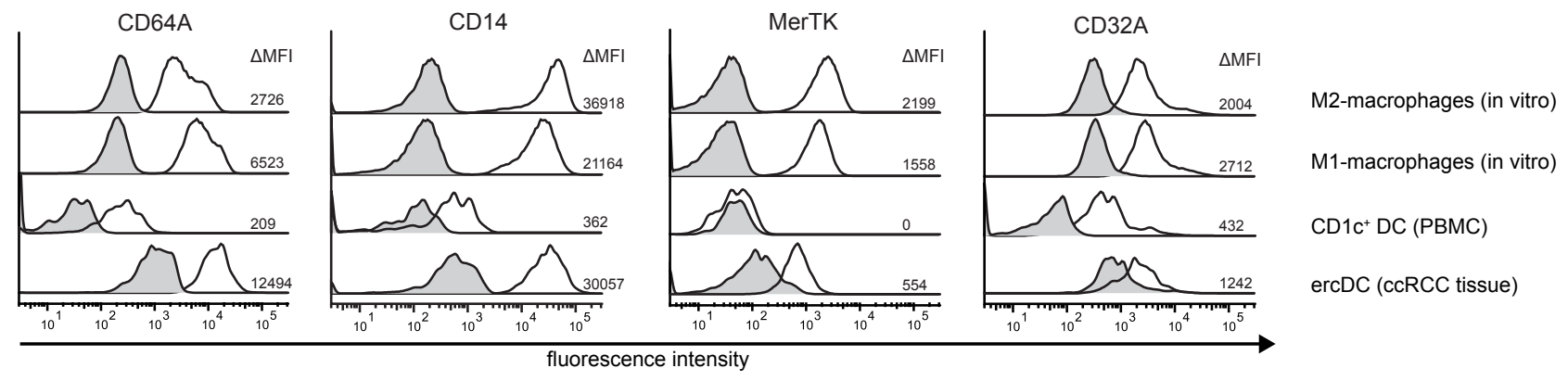

C

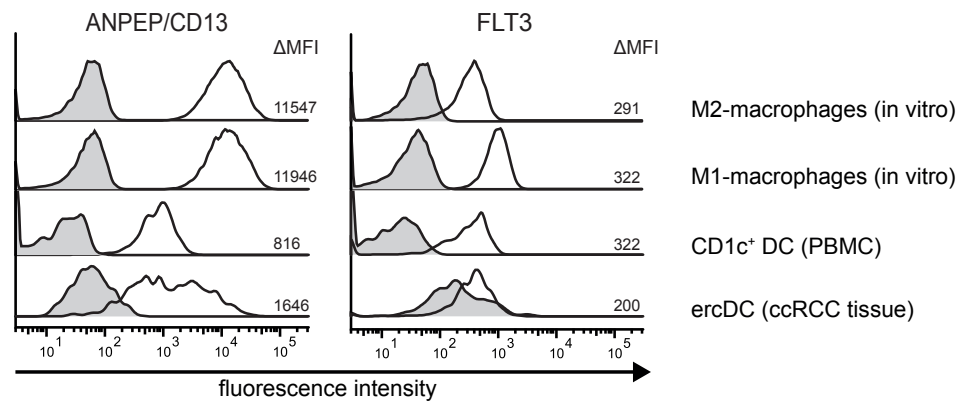




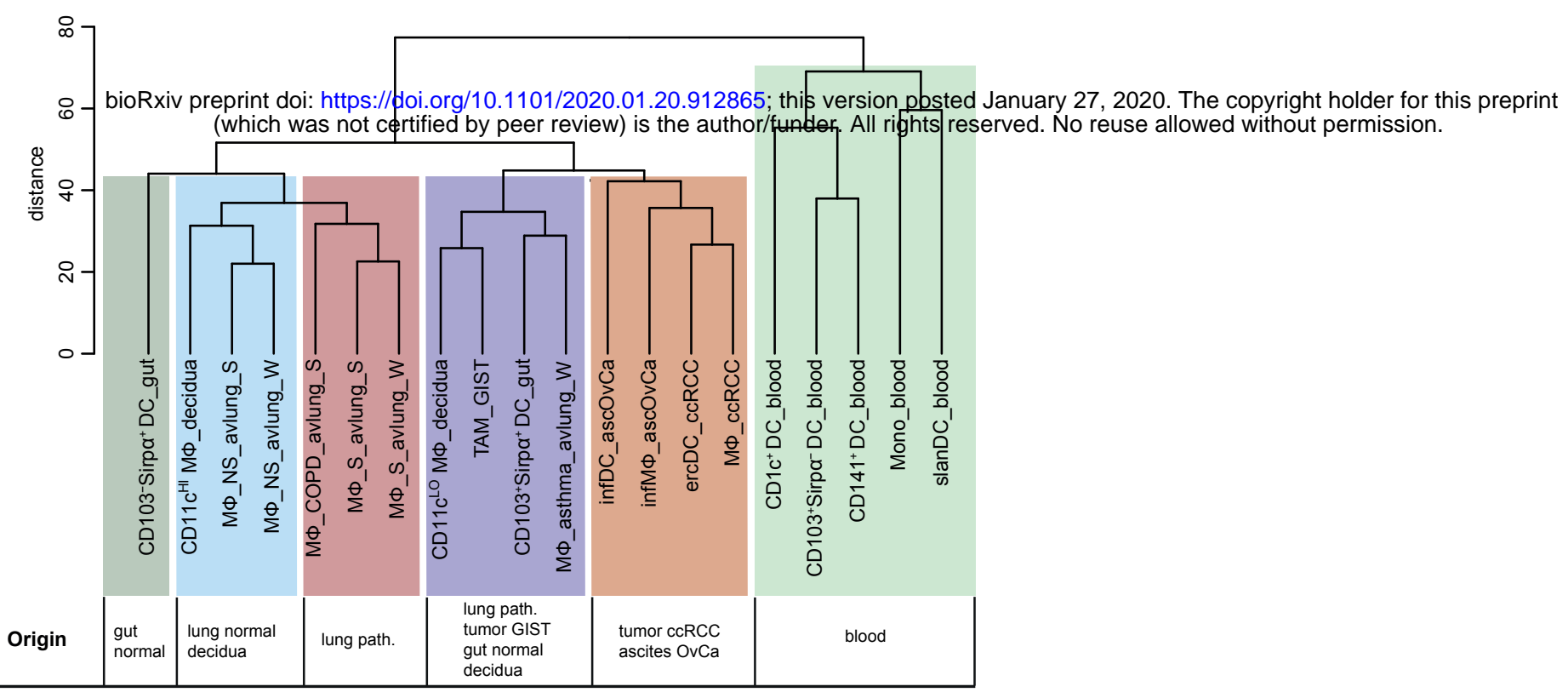

B

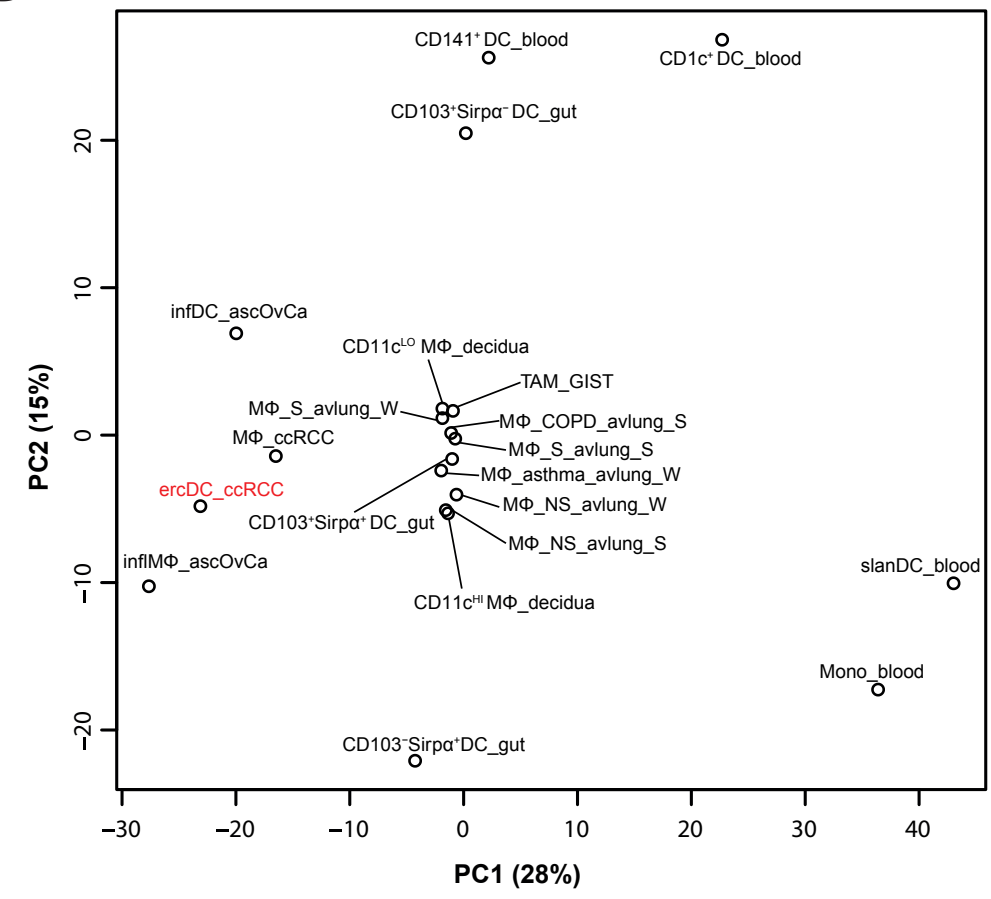


A
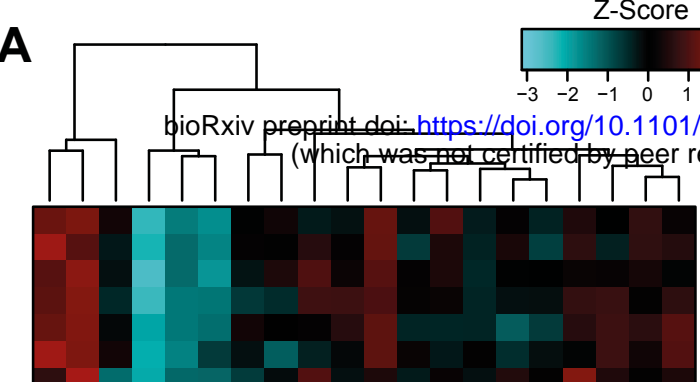

APOE

APOC1

GPNMB

FABP3

SLC38A6

CCL18

ADAMDEC1

IL2RA

RHOBTB3

NRP1

NRP2

DAB2

LGMN

CCL8

ME1

MAF

GAL3ST4

FOLR2

SDC3

SEPP1

ABCC5

CRYAB

SERPING1

SIGLEC 1

C1QA

SLCO2B1

C1QB

C2

PLTP

IGF1

ARHGAP12

CD209

MS4A $4 A$

PLXDC1

SCAMP5

ANKRD36

CXCL9

IRAK3

LILRA1

EMR3

YYST

LYS

CD52

CD52

PLAC8

S100A12

FCN1

TES

FYN

FLNA

FGR

CDC42EP3

FAM65B

CCDC88C

CD48

ACPP

CAPN2

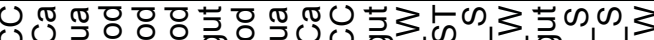

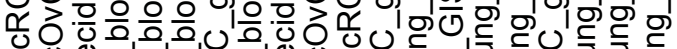

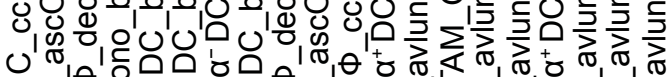

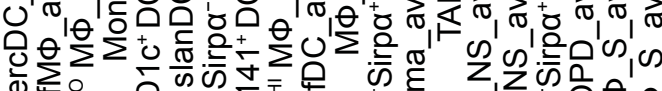

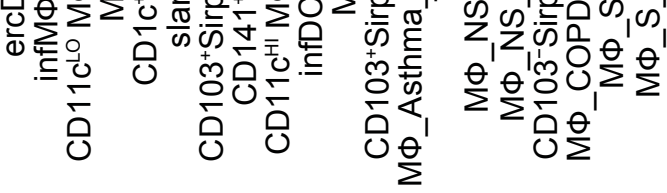

B

APOC1 FOLR2

SDC3 GAL3ST4

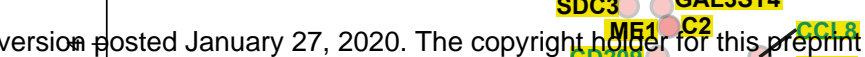

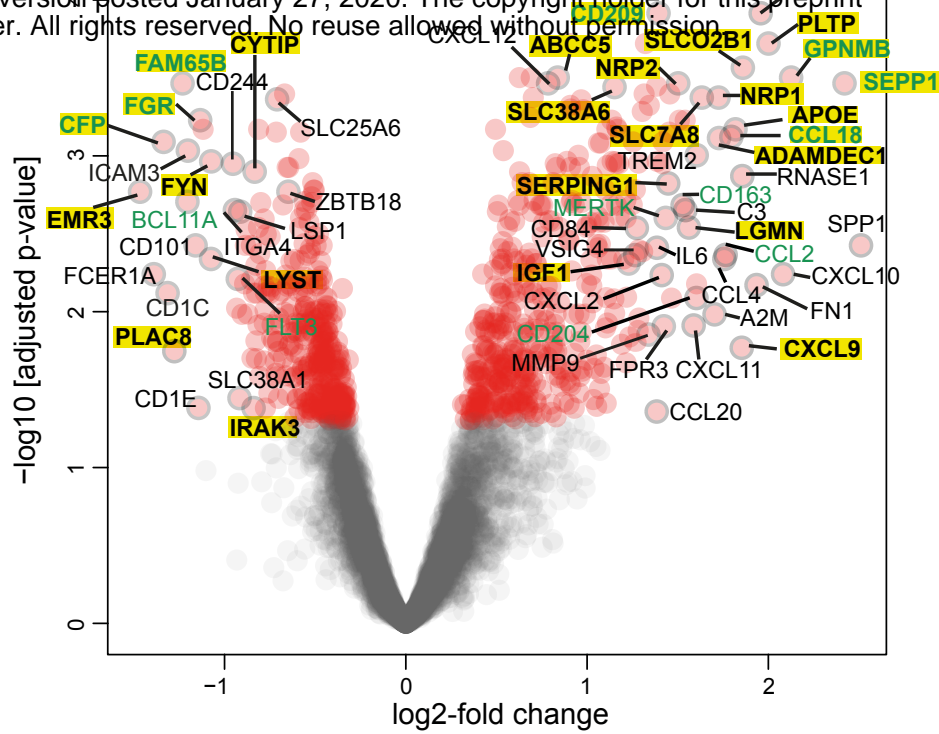

ercDC_ccRCC \& infM $\Phi$ _ascOvCa vs. control group

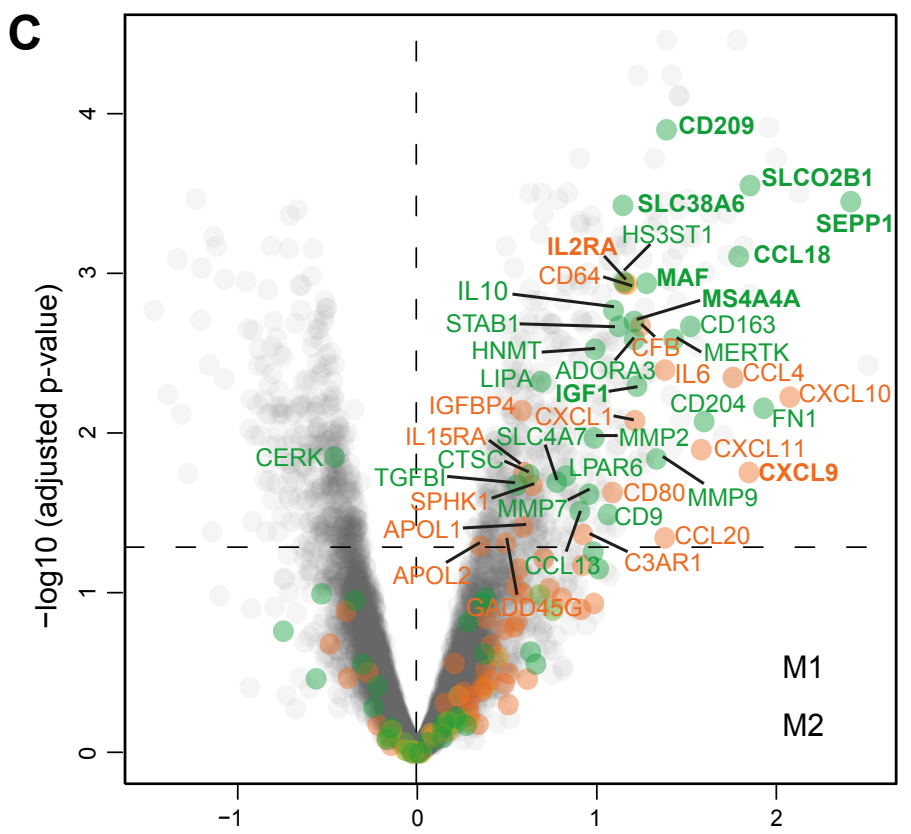

log2-fold change

ercDC_ccRCC \& infMФ_ascOvCa vs. control group

\begin{tabular}{|l|l|l|}
\hline & M1 genes & M2 genes \\
\hline Informative & 76 & 62 \\
\hline ercDC_ccRCC DEGs & $18(23.7 \%)$ & $28(45.2 \%)$ \\
\hline ercDC_ccRCC marker & $2(2.6 \%)$ & $8(12.9 \%)$ \\
\hline
\end{tabular}


Response to wounding Inflammatory response

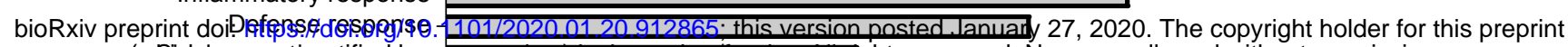

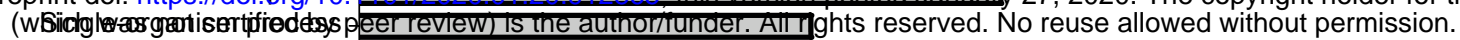

Response to stress

Regulation of response to external stimulus Immune system process

Regulation of defense response Localization

Locomotion

Translational initiation

Complement activation Cell migration

Regulation of immune effector process Regulation of inflammatory response Acute inflammatory response Humoral immune response Response to stimulus Single-multicellular organism process
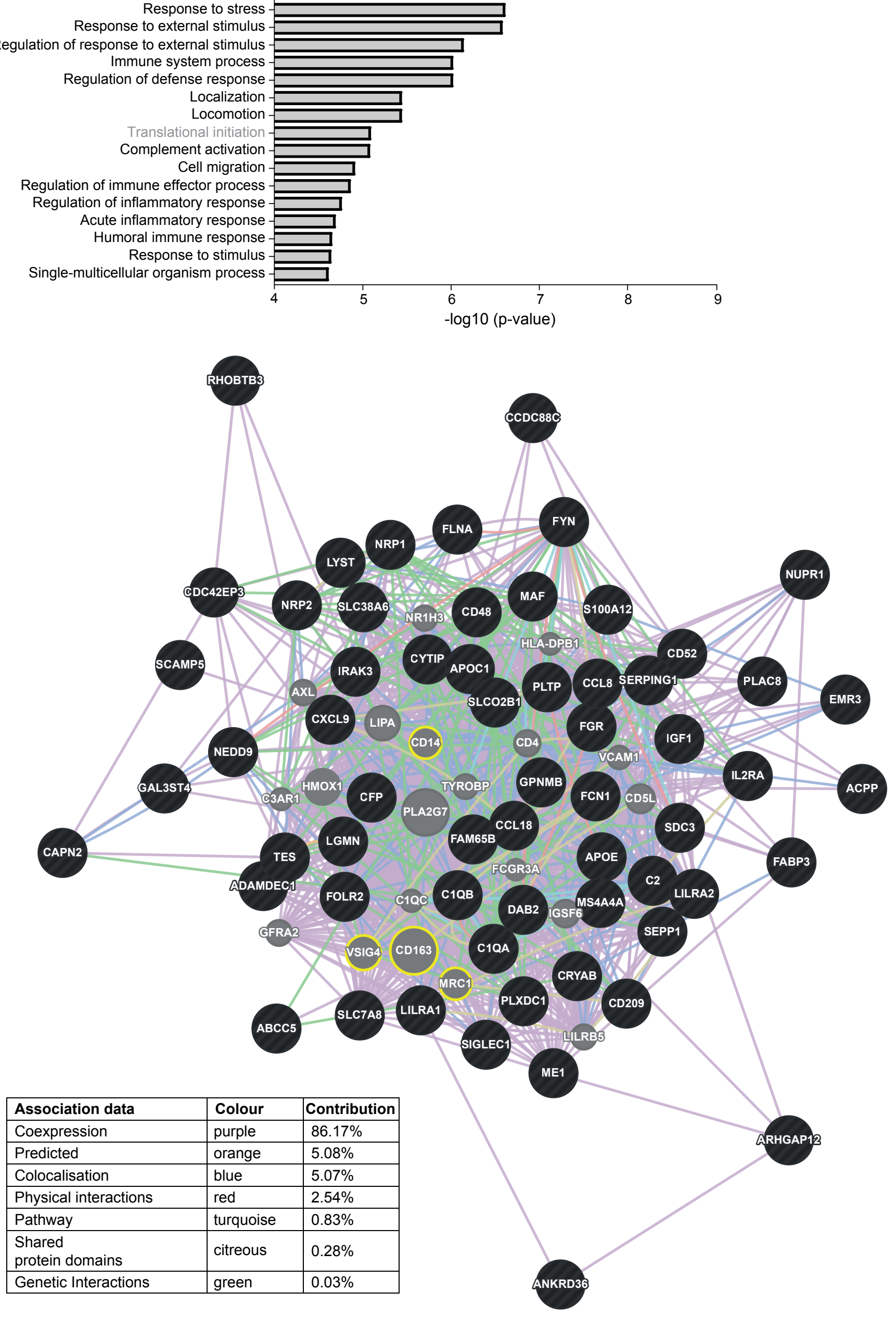
A

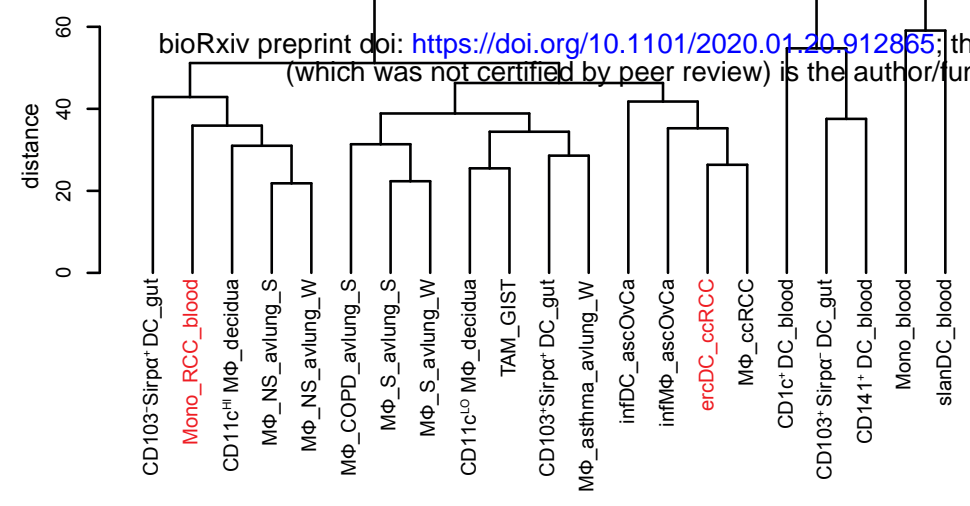

B

CD141+DC_blood CD1c ${ }^{+}$DC_blood

$\mathrm{CD}_{103^{+}} \mathrm{Sirpa}^{-} \mathrm{DC}$ gut
The copyright holder for this preprint this version posted January 27,2020 . The copyright holder for this
under. All rights reserved. No reuse allowed without permission.

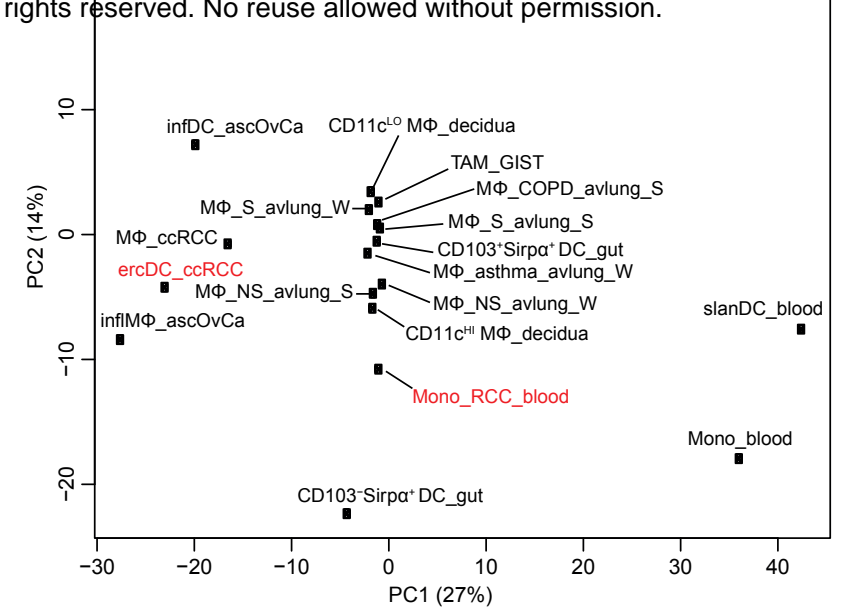

C

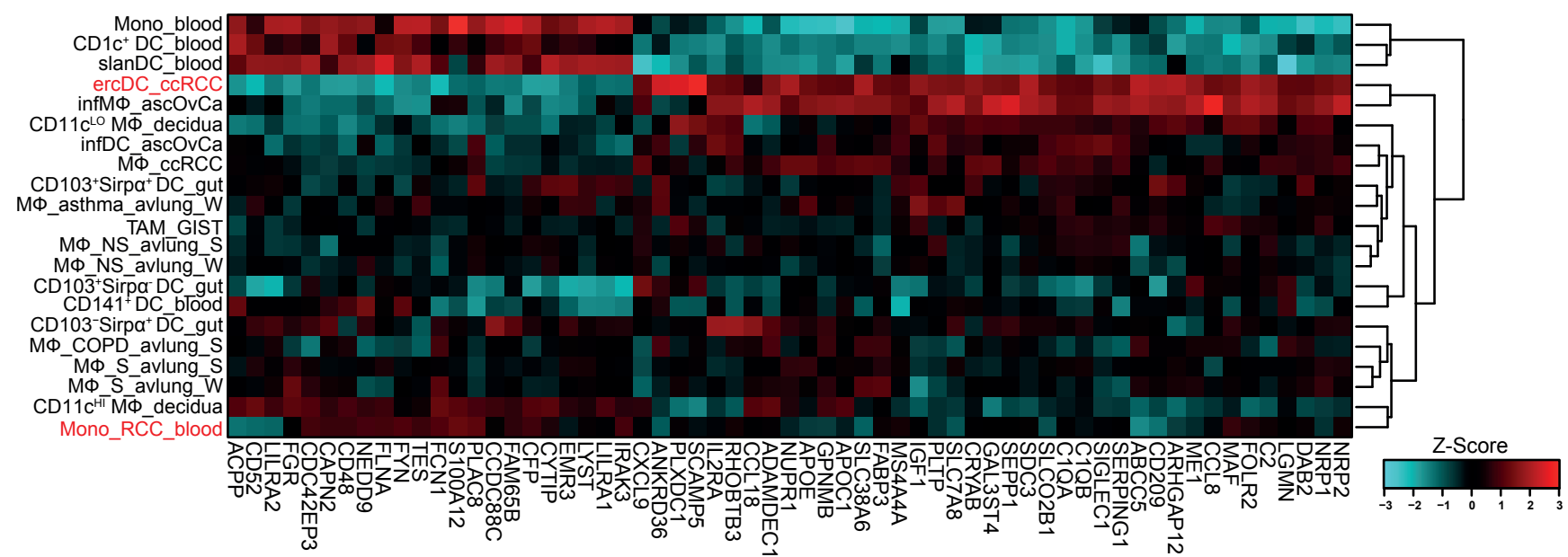

D

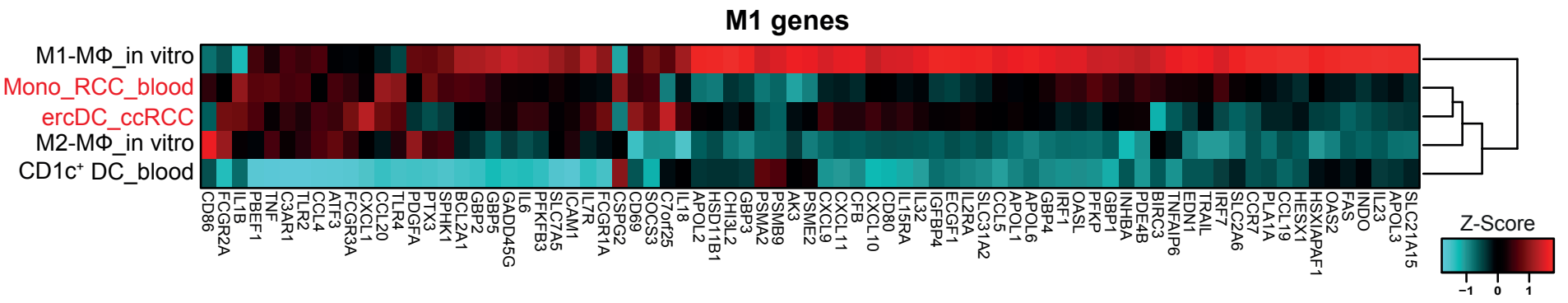

M2 genes

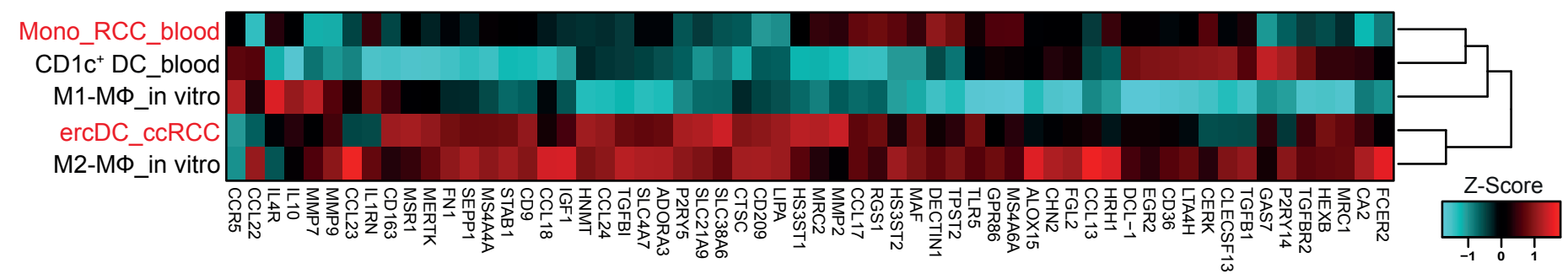

E

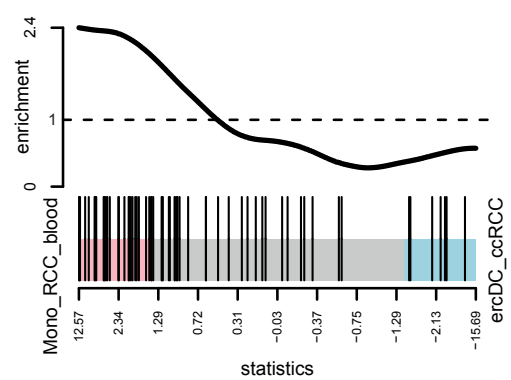


A

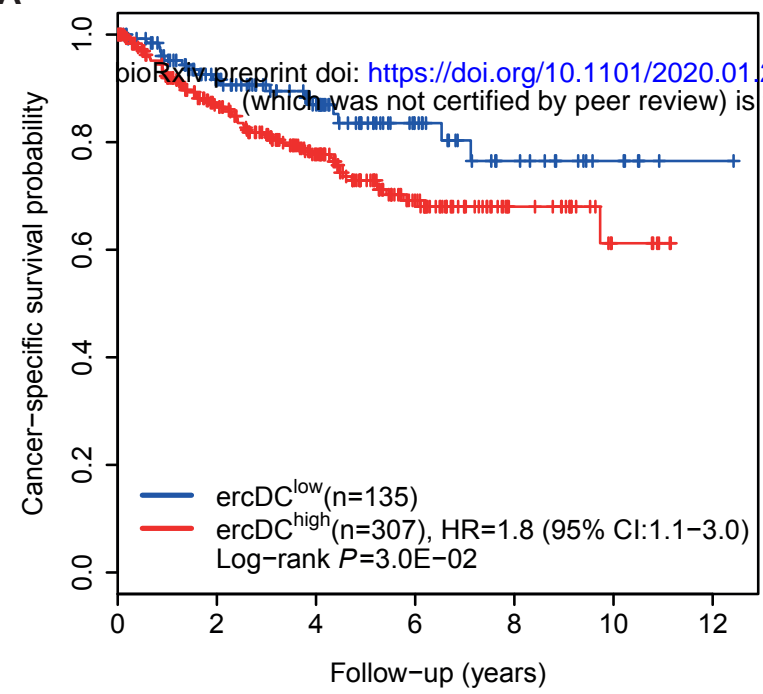

C

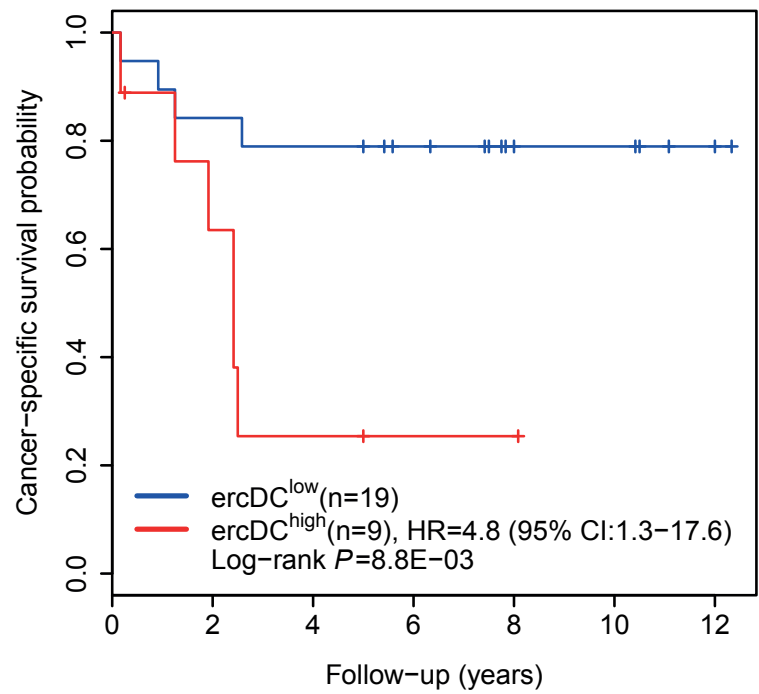

B

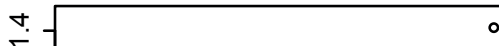

○

65 ; this version posted January $2 \boldsymbol{2}, 2020$. The copyright holpler for this preprint

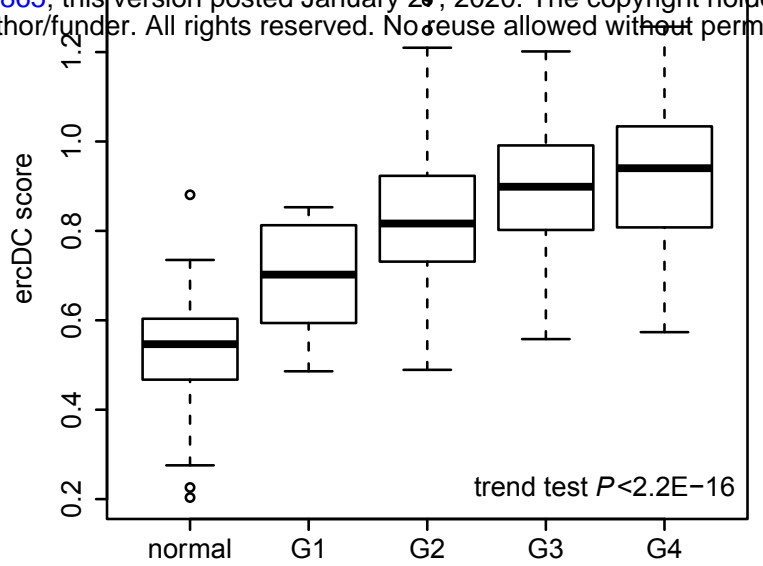

D

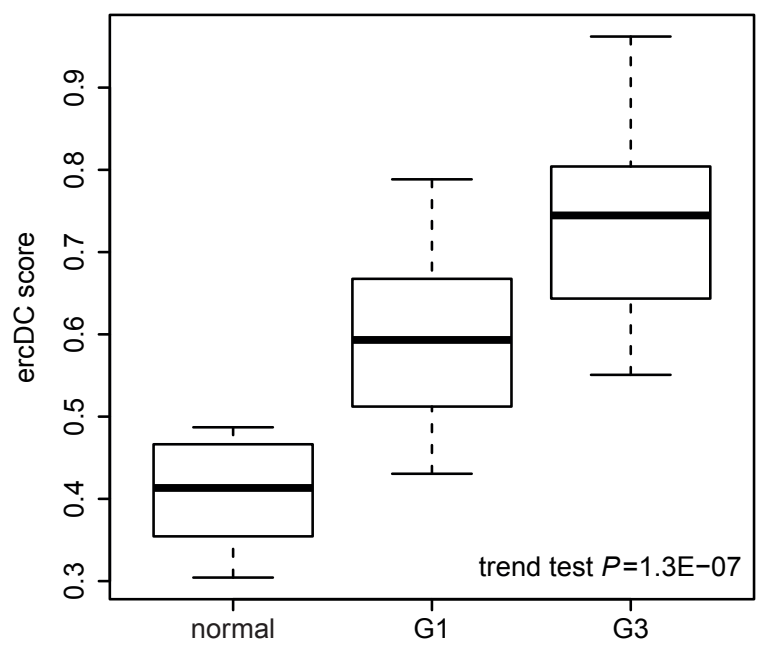

E

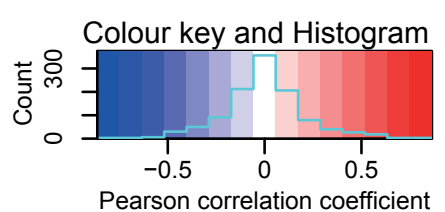

Pearson correlation coefficient
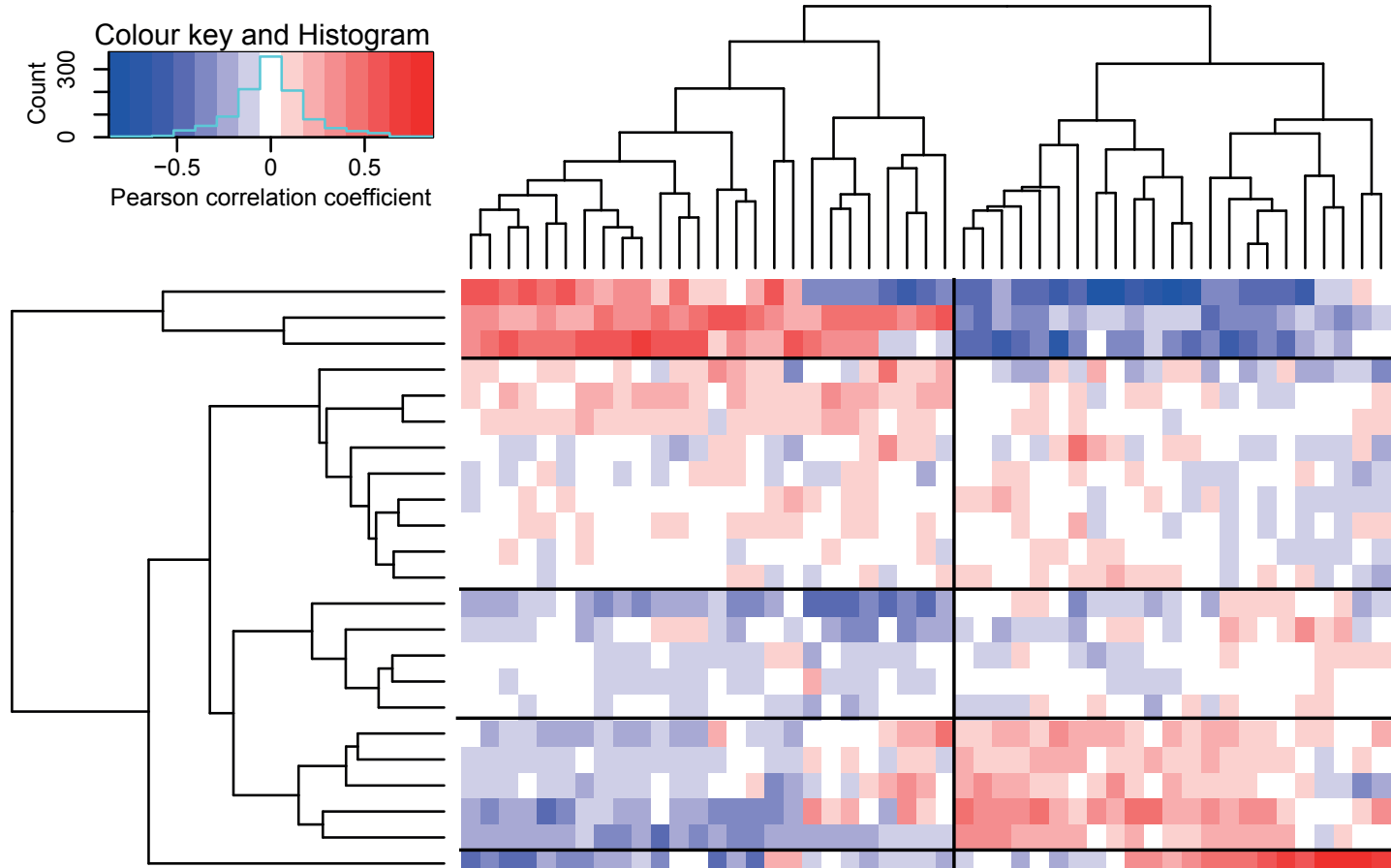

Cell type

group

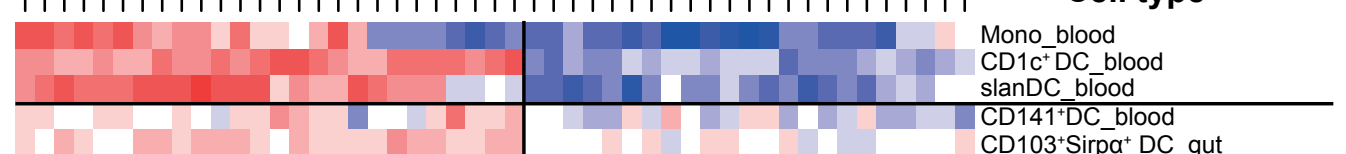

CD141+DC_blood

CD103 Sirpa DC_gut

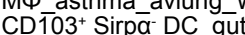

MФ COPD avlung S

TAM GIST $C D 11 c^{\mathrm{LO}} \mathrm{M \Phi} \Phi_{-}$decidua

MФSS_avlung_S

MФ ${ }^{-}$- avlung $W$

CD103-Sirpa ${ }^{+}$DC_gut

MФ NS avlung $S$

M $\Phi^{-}$NS - avlung_W

$\mathrm{CD} 11 \mathrm{C}^{\mathrm{H}} \mathrm{M} \Phi$ decidua

infDC_ascOvCa

$\mathrm{M} 2-\mathrm{M} \Phi$ in vitro

$M \Phi$ ccRCC infM $\Phi$ ascOvCa

ercDC $\mathrm{ccRCC}$

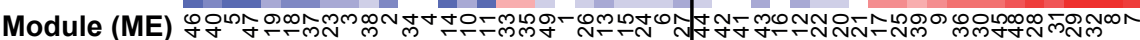

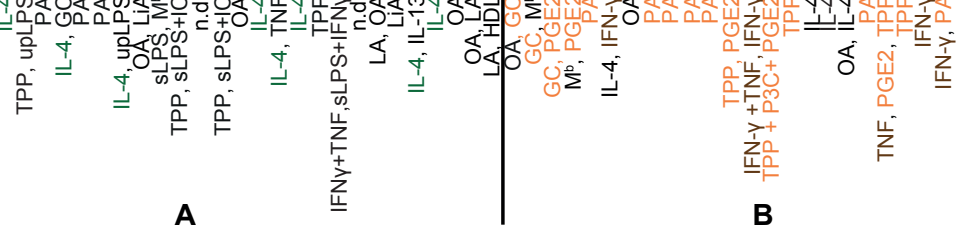


A

VSIG4 [transcript]

B

VSIG4 [surface protein]

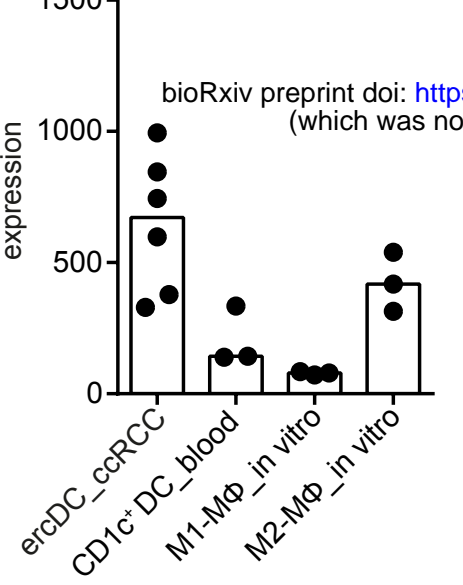

$\triangle \mathrm{MFI}$

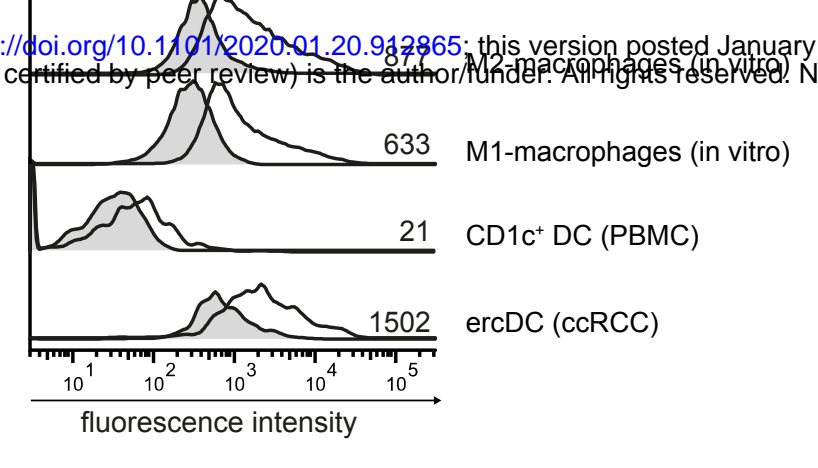

E
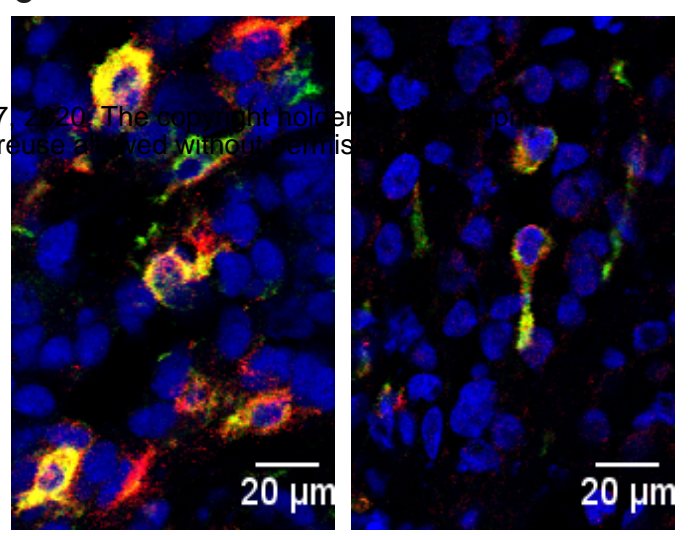

- CD209

- VSIG4

- DAPI
D
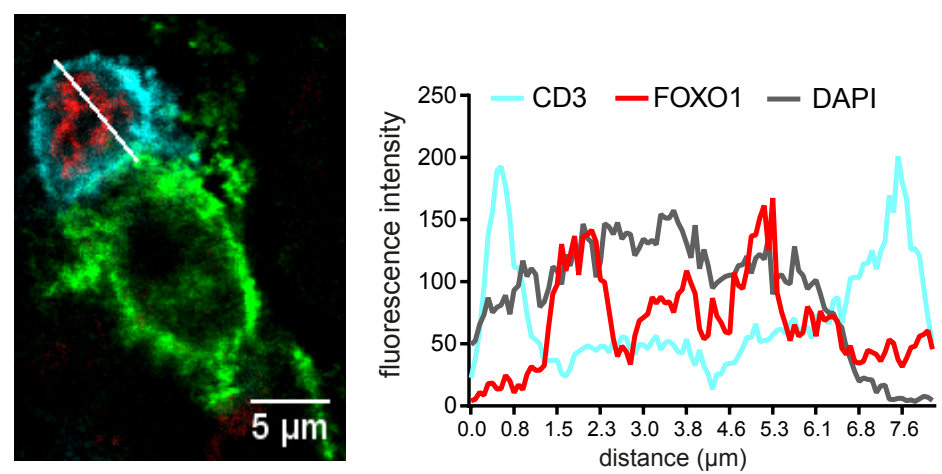

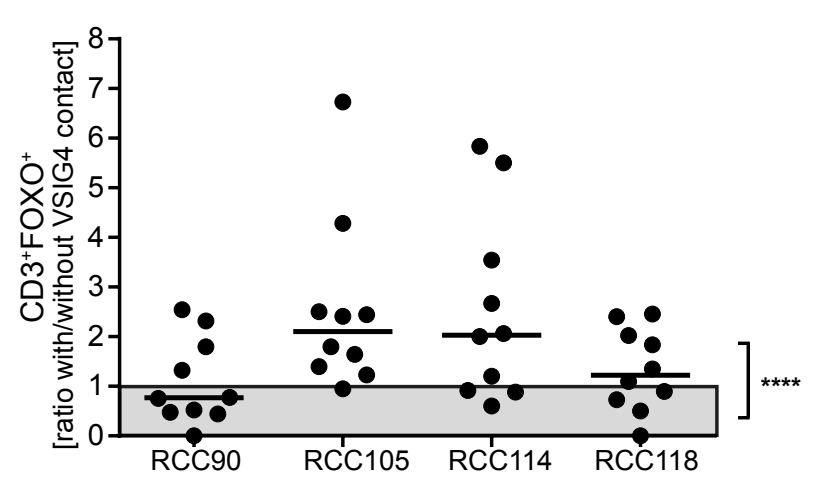

Figure 10 